\title{
Complex Atanassov's Intuitionistic Fuzzy Relation
}

\author{
Abd Ulazeez M. Alkouri and Abdul Razak Salleh \\ School of Mathematical Sciences, Faculty of Science and Technology, Universiti Kebangsaan Malaysia, \\ 43600 UKM Bangi, Selangor, Malaysia \\ Correspondence should be addressed to Abd Ulazeez M. Alkouri; abd.aziz986@yahoo.com
}

Received 21 January 2013; Revised 22 April 2013; Accepted 24 April 2013

Academic Editor: Juan P. Rincón-Zapatero

Copyright (C) 2013 A. U. M. Alkouri and A. R. Salleh. This is an open access article distributed under the Creative Commons Attribution License, which permits unrestricted use, distribution, and reproduction in any medium, provided the original work is properly cited.

\begin{abstract}
This paper presents distance measure between two complex Atanassov's intuitionistic fuzzy sets (CAIFSs). This distance measure is used to illustrate an application of CAIFSs in solving one of the most core application areas of fuzzy set theory, which is multiattributes decision-making (MADM) problems, in complex Atanassov's intuitionistic fuzzy realm. A new structure of relation between two CAIFSs, called complex Atanassov's intuitionistic fuzzy relation (CAIFR), is obtained. This relation is formally generalised from a conventional Atanassov's intuitionistic fuzzy relation, based on complex Atanassov's intuitionistic fuzzy sets, in which the ranges of values of CAIFR are extended to the unit circle in complex plane for both membership and nonmembership functions instead of $[0,1]$ as in the conventional Atanassov's intuitionistic fuzzy functions. Definition and some mathematical concepts of CAIFS, which serve as a foundation for the creation of complex Atanassov's intuitionistic fuzzy relation, are recalled. We also introduce the Cartesian product of CAIFSs and derive two properties of the product space. The concept of projection and cylindric extension of CAIFRs are also introduced. An example of CAIFR in real-life situation is illustrated in this paper. Finally, we introduce the concept of composition of CAIFRs.
\end{abstract}

\section{Introduction}

The idea of the concept of Atanassov's intuitionistic fuzzy set (AIFS) was introduced by Atanassov [1], where he achieved his concept by adding the nonmembership term to the definition of fuzzy set (FS) that was given by Zadeh [2], while the fuzzy set has only one component, a membership function. It is well known that the range of each membership and nonmembership functions is limited to $[0,1]$, where it belongs to the real numbers. In 2007, Fathi [3] introduced some operations on AIFSs called $S$-norm and $T$-norm. The question presented by Daniel Ramot and other researchers was that what will be the result if we change the codomain of the fuzzy sets to complex numbers? In 2002, Ramot et al. [4] introduced a new innovative concept which they called a complex fuzzy set (CFS). On the other hand, complex fuzzy set has been used to solve various problems, in particular the multiple periodic factor prediction problems [5]. A neurofuzzy system architecture implementing complex fuzzy rule was defined by Chen et al. [6], which is the first practical application of the concept of complex fuzzy logic.
Atanassov's intuitionistic fuzzy set has several applications in many areas and has been used to solve multiple attributes decision making (MADM) problems, see [7-10]. Atanassov's intuitionistic fuzzy set represents the information involving two or more answers of type: yes, no, I do not know, I am not sure, and so forth. Our purpose in this paper is to give an example of application that represents this type of information which is happening repeatedly over a period of time. In our previous conference paper [11], we introduced the concept of complex Atanassov's intuitionistic fuzzy set. Complex Atanassov's intuitionistic fuzzy set can represent the information on people's decision which happens periodically. In addition, we also represent the uncertainty of complexvalued functions in many physical quantities, for instance, wave function in quantum mechanics, impedance in electrical engineering, complex amplitude, and so forth. We can also define the values of belongingness and nonbelongingness for any object in these complex-valued functions.

In the literature $[4,11]$, the novelty of using complex numbers appears in the phase term to get more rangevalued membership functions to represent the problems with 
uncertainty and periodicity simultaneously. In addition, it indicates the uncertainty of complex-valued functions in many physical quantities, for instance, wave function in quantum mechanics, impedance in electrical engineering, complex amplitude, and so forth. In this paper, the novelty also appears in the phase term but for both membership and nonmembership functions in some inherent concepts, in contrast to CFS which is only characterized by membership function. The literature novelties of phase term are extended to appear in several prominent concepts under the case of CAIFS, for instance, distance measure, Cartesian product, relations, projection, cylindric extension, and so forth. The CFS [4] has only one additional phase term, but in CAIFS [11], we have two additional phase terms. This confers more range values to represent the uncertainty and periodicity semantics simultaneously, and to define the values of belongingness and nonbelongingness for any object in these complex-valued functions. Undoubtedly, the property of periodic nature of complex-valued memberships helps us to introduce the present concepts. In other words, the periodicity semantic can be denoted as a phase term in complex numbers, and the uncertainty semantic can be denoted as an amplitude term in complex numbers.

The difference between CAIFS and AIFS is that in our concept, CAIFS has an ability to represent the problems with Atanassov's intuitionistic uncertainty and periodicity simultaneously. The phase term of CAIFS shows that at least in some cases a second dimension (periodic dimension) of membership is required, where the phase term is the distinguishing factor between AIFS and CAIFS. In this paper, we employ CAIFS structure in MADM problems to choose the best alternative by generalising the selection model from [12]. It is worth noting that one of the most useful properties of the phase term is the ability to convey Atanassov's intuitionistic fuzzy information.

It should be noted that there are many researchers who combined complex numbers and fuzzy sets, such as Buckley [13], Nguyen et al. [14], and Zhang et al. [15, 16]. On the other hand, Ramot et al. [4] introduced a new approach that is absolutely different from them, where they extended the range of membership function to unit circle in the complex plane, unlike the others who limited the range to $[0,1]$. Ramot et al. [4] also added an additional term called the phase term to solve the enigma in translating some complex-valued functions on physical terms to human language and vice versa. In 2011, Jun et al. [5] employed complex fuzzy sets to represent the information with uncertainty and periodicity, where they introduced a product-sum aggregation operator(PSAO-) based prediction (PSAOP) method to generate a solution of multiple periodic factor prediction (MPFP) problems.

Since 1965, the concept of fuzzy set theory was introduced by Zadeh [2]; numerous researchers did intensive studies on fuzzy relation (simply denoted by FR) such as Di Nola et al. $[17,18]$ and Bandler and Kohout [19]. According to Mendel [20] in 1995 "fuzzy relation represents a degree of presence or absence of association, interaction, or interconnectedness" between the elements of two or more sets. Later in 2002, complex fuzzy relation (CFR) was introduced by Ramot et al. [4], where "the CFRs represent both the degree of presence or absence of association, interaction, or interconnectedness, and the phase of association, interaction, or interconnectedness between the elements of two or more sets."

Burillo and Bustince introduced the definition of Atanassov's intuitionistic fuzzy relation and studied some of its properties [21-24]. In 2010, Atanassov's intuitionistic fuzzy relation was reintroduced by Adam [25] based on the definition of Atanassov's intuitionistic fuzzy relation given by Bustince and Burillo. Adam generalised fuzzy relation to Atanassov's intuitionistic fuzzy relation by adding the nonmembership functions to the fuzzy relation structure. In this paper, we introduce CAIFRs by extending each value of the membership and nonmembership functions from real number, $[0,1]$, to complex number, the unit circle. The main benefit expected to be gained from the introduction of the concept of CAIFR is the ability to denote both the presence and absence of association, interaction, or interconnectedness in one set of CAIFS instead of two sets as in CFS, where CAIFS has membership and nonmembership functions in the same set, whilst CFS contains only the membership functions and denotes the presence or absence of association, interaction, or interconnectedness.

\section{Preliminaries}

In this section, we recall some definitions and results that will be effectively employed in this paper.

Definition 1 (see [2]). A fuzzy set $A$ in a universe of discourse $X$ is characterized by a membership function $\mu_{A}(x)$ that takes values in the interval $[0,1]$.

Definition 2 (see [1]). An Atanassov's intuitionistic fuzzy set (AIFS) $A$ in a nonempty set $X$ (a universe of discourse) is an object having the following form:

$$
A=\left\{\left\langle x, \mu_{A}(x), \gamma_{A}(x)\right\rangle: x \in X\right\},
$$

where the functions $\mu_{A}(x): X \rightarrow[0,1], \gamma_{A}(x): X \rightarrow$ $[0,1]$ denote the degree of membership and degree of nonmembership of each element $x \in X$ to the set $A$, respectively, and $0 \leq \mu_{A}(x)+\gamma_{A}(x) \leq 1$ for all $x \in X$.

Definition 3 (see [26]). The complement, union, and intersection of two AIFSs $A=\left\{\left\langle x, \mu_{A}(x), \gamma_{A}(x)\right\rangle: x \in X\right\}$ and $B=\left\{\left\langle x, \mu_{B}(x), \gamma_{B}(x)\right\rangle: x \in X\right\}$, in a universe of discourse $X$, are defined as follows:
(a) $\bar{A}=\left\{\left\langle x, \gamma_{A}(x), \mu_{A}(x)\right\rangle: x \in X\right\}$,
(b) $A \cup B=\left\{\left\langle x, \max \left(\mu_{A}(x), \mu_{B}(x)\right), \min \left(\gamma_{A}(x), \gamma_{B}(x)\right)\right\rangle\right.$ : $x \in X\}$,
(c) $A \cap B=\left\{\left\langle x, \min \left(\mu_{A}(x), \mu_{B}(x)\right), \max \left(\gamma_{A}(x), \gamma_{B}(x)\right)\right\rangle\right.$ : $x \in X\}$.

Definition 4 (see [4]). A complex fuzzy set (CFS) A, defined on a universe of discourse $X$, is characterized by membership functions $\mu_{A}(x)$ that assign to any element $x \in X$ a complexvalued grade of membership in $A$. 
By definition, the values of $\mu_{A}(x)$ may receive all lying within the unit circle in the complex plane and are thus of the form $\mu_{A}(x)=r_{A}(x) \cdot e^{i \omega_{A}(x)}$, where $i=\sqrt{-1}$, each of $r_{A}(x)$ and $\omega_{A}(x)$ is real-valued, and $r_{A}(x) \in[0,1]$. The CFS $A$ may be represented as the set of ordered pairs $A=\left\{\left(x, \mu_{A}(x)\right)\right.$ : $x \in X\}$.

Definition 5 (see [25]). Let $A_{1}, \ldots, A_{n}$ be Atanassov's intuitionistic fuzzy sets in $X_{1}, \ldots, X_{n}$, respectively. The Cartesian product $A_{1} \times \cdots \times A_{n}$ is an Atanassov's intuitionistic fuzzy set defined by

$$
\begin{aligned}
& A_{1} \times \cdots \times A_{n} \\
& =\left\{\left\langle\left(x_{1}, \ldots, x_{n}\right), \mu_{A_{1} \times \cdots \times A_{n}}\left(x_{1}, \ldots, x_{n}\right),\right.\right. \\
& \left.\left.\quad \gamma_{A_{1} \times \cdots \times A_{n}}\left(x_{1}, \ldots, x_{n}\right)\right\rangle:\left(x_{1}, \ldots, x_{n}\right) \in\left(X_{1}, \ldots, X_{n}\right)\right\},
\end{aligned}
$$

where $\mu_{A_{1} \times \cdots \times A_{n}}\left(x_{1}, \ldots, x_{n}\right)=\min \left[\mu_{A_{1}}\left(x_{1}\right), \ldots, \mu_{A_{n}}\left(x_{n}\right)\right]$, and $\gamma_{A_{1} \times \cdots \times A_{n}}\left(x_{1}, \ldots, x_{n}\right)=\max \left[\gamma_{A_{1}}\left(x_{1}\right), \ldots, \gamma_{A_{n}}\left(x_{n}\right)\right]$.

Definition 6 (see [25]). Let $Q$ be an Atanassov's intuitionistic fuzzy relation in $X_{1} \times \cdots \times X_{n}$, and let $\left\{i_{1}, \ldots, i_{k}\right\}$ be a subsequence of $\{1,2, \ldots, n\}$. Then the projection of $Q$ on $X_{i_{1}} \times \cdots \times X_{i_{k}}$ is an Atanassov's intuitionistic fuzzy relation $Q_{p}$ on $X_{i_{1}} \times \cdots \times X_{i_{k}}$, defined by membership and nonmembership functions,

$$
\begin{aligned}
& \mu_{Q_{p}}\left(x_{i_{1}}, \ldots, x_{i_{k}}\right)=\max _{x_{j_{1}} \in X_{j_{1}}, \ldots, x_{j_{(n-k)}} \in X_{j_{(n-k)}}} \mu_{\mathrm{Q}}\left(x_{1}, \ldots, x_{n}\right), \\
& \gamma_{\mathrm{Q}_{p}}\left(x_{i_{1}}, \ldots, x_{i_{k}}\right)=\min _{x_{j_{1}} \in X_{j_{1}}, \ldots, x_{j_{(n-k)}} \in X_{j_{(n-k)}}} \gamma_{\mathrm{Q}}\left(x_{1}, \ldots, x_{n}\right),
\end{aligned}
$$

where the $\left\{x_{j_{1}}, x_{j_{2}}, \ldots, x_{j_{(n-k)}}\right\}$ is the complement of $\left\{x_{i_{1}}, x_{i_{2}}, \ldots, x_{i_{k}}\right\}$ with respect to $\left\{x_{1}, x_{2}, \ldots, x_{n}\right\}$.

Definition 7 (see [25]). Let $Q$ be an Atanassov's intuitionistic fuzzy relation in $X_{1} \times \cdots \times X_{n}$, and let $\left\{i_{1}, \ldots, i_{k}\right\}$ be a subsequence of $\{1,2, \ldots, n\}$, then the cylindric extension of $Q_{p}$ to $X_{1} \times \cdots \times X_{n}$ is an Atanassov's intuitionistic fuzzy relation $Q_{\mathrm{PE}}$ on $X_{1} \times \cdots \times X_{n}$, defined by membership and nonmembership functions:

$$
\begin{aligned}
& \mu_{\mathrm{QPE}_{\mathrm{PE}}}\left(x_{1}, \ldots, x_{n}\right)=\mu_{\mathrm{Q}_{P}}\left(x_{i_{1}}, \ldots, x_{i_{n}}\right), \\
& \gamma_{\mathrm{QPE}_{\mathrm{PE}}}\left(x_{1}, \ldots, x_{n}\right)=\gamma_{\mathrm{Q}_{P}}\left(x_{i_{1}}, \ldots, x_{i_{n}}\right) .
\end{aligned}
$$

Definition 8 (see [4]). Let $X$ and $Y$ be two sets. A complex fuzzy relation $R$ of $X$ and $Y$, written $R(U, V)$, is a complex fuzzy subset of the product space $X \times Y$. The relation $R(X, Y)$ is characterized by the complex membership function $\mu_{R}(x, y)$, where $x \in X$ and $y \in Y$, and $\mu_{R}(x, y)$ assigns to each pair $(x, y)$ a complex-valued grade of membership to the set $R(X, Y)$. As always, $R(X, Y)$ may be represented as the set of ordered pairs:

$$
R(X, V)=\left\{\left((x, y), \mu_{R}(x, y)\right) \mid(x, y) \in X \times Y\right\} .
$$

The values $\mu_{R}(x, y)$ may be took from the unit circle in the complex plane and are of the following form: $r(x) \cdot e^{i \omega(x)}(i=$ $\sqrt{-1}), r(x)$ and $\omega(x)$ are both real-valued, with $r(x) \in[0,1]$.
The complex membership function is to be interpreted in the following manner:

(i) $r(x)$ represents a degree of presence or absence of association, interaction, or interconnectedness between the elements of $X$ and $Y$;

(ii) $\omega(x)$ represents the phase of association, interaction, or interconnectedness between the elements of $X$ and $Y$.

Definition 9 (see [15]). Let $X, Y$, and $Z$ be universes, $A$ a complex fuzzy relation of $X$ and $Y$, and $B$ a complex fuzzy relation of $Y$ and $Z$. Then, we say a composition of $A$ and $B$, denoted by $A \circ B$, is a complex fuzzy relation of $X$ and $Z$, is specified by the following function

$$
\begin{aligned}
\mu_{A \circ B}(x, z)= & r_{A \circ B}(x, z) \cdot e^{i \arg _{A \bullet B}(x, z)} \\
= & \sup _{y \in Y} \min \left(r_{A}(x, y), r_{B}(y, z)\right) \\
& \cdot e^{i \sup _{y \in Y} \min \left[\arg _{A}(x, y), \arg _{B}(y, z)\right]} .
\end{aligned}
$$

Example 10 (see [15]). Let

$$
\begin{gathered}
A(X, Y)=\left(\begin{array}{ccc} 
& y_{1} & y_{2} \\
x_{1} & 0.6 e^{i 1.2 \pi} & 0.8 e^{i 1.6 \pi} \\
x_{2} & 1.0 e^{i 2 \pi} & 0.5 e^{i \pi}
\end{array}\right), \\
B(Y, Z)=\left(\begin{array}{ccc}
z_{1} & z_{2} \\
y_{1} & 0.6 e^{i 1.2 \pi} & 0.8 e^{i 1.6 \pi} \\
y_{2} & 1.0 e^{i 2 \pi} & 0.8 e^{i 1.6 \pi}
\end{array}\right)
\end{gathered}
$$

be two relational matrices. Then, to compute $A \circ B(X, Z)$, we should note that $X \times Z$ has four elements: $A \circ B=$ $\left(\begin{array}{l}\left(x_{1}, z_{1}\right)\left(x_{1}, z_{2}\right) \\ \left(x_{2}, z_{1}\right)\end{array}\left(x_{2}, z_{2}\right)\right.$. Thus, our task is to determine the membership, $\mu_{A \circ B}$, values for the four elements. For instance, we use the function in Definition 9 to determine the membership function of $A \circ B\left(x_{1}, z_{1}\right)$, so we have

$$
\begin{aligned}
& A \circ B\left(x_{1}, z_{1}\right) \\
& =\sup _{y \in Y} \min \left(r_{A}\left(x_{1}, y\right), r_{B}\left(y, z_{1}\right)\right) \\
& \cdot e^{i \sup _{y \in Y} \min \left(\omega_{A}\left(x_{1}, y\right), \omega_{B}\left(y, z_{1}\right)\right)} \\
& =\sup _{y \in Y}\left\{\min \left[\left(r_{A}\left(x_{1}, y_{1}\right)\right), r_{B}\left(y_{1}, z_{1}\right)\right],\right. \\
& \left.\min \left[\left(r_{A}\left(x_{1}, y_{2}\right)\right), r_{B}\left(y_{2}, z_{1}\right)\right]\right\} \\
& \cdot e^{\sup _{y \in Y}\left\{\min \left[\left(\omega_{r_{A}}\left(x_{1}, y_{1}\right)\right), \omega_{r_{B}}\left(y_{1}, z_{1}\right)\right], \min \left[\left(\omega_{r_{A}}\left(x_{1}, y_{2}\right)\right), \omega_{r_{B}}\left(y_{2}, z_{1}\right)\right]\right\}} \\
& =\sup _{y \in Y}\{\min [0.6,0.6], \min [0.8,1]\} \\
& \cdot e^{i \sup _{y \in Y}\{\min [1.2 \pi, 1.2 \pi], \min [1.6 \pi, 2 \pi]\}} \\
& =\sup _{y \in Y}\{0.6,0.8\} \cdot e^{i \sup _{y \in Y}\{1.2 \pi, 1.6 \pi\}}=0.8 \cdot e^{i 1.6 \pi} .
\end{aligned}
$$


Analogously, we determine the elements $\left(x_{1}, z_{2}\right)$, $\left(x_{2}, z_{1}\right)$, and $\left(x_{2}, z_{2}\right)$ therefore, the final relational matrix of $A \circ B$ is

$$
A \circ B=\left(\begin{array}{ll}
0.8 e^{i 1.6 \pi} & 0.6 e^{i 1.2 \pi} \\
0.8 e^{i 1.6 \pi} & 0.8 e^{i 1.6 \pi}
\end{array}\right) .
$$

Proposition 11 (see [15]). Let $W, X, Y$, and $Z$ be universes, $A$ a complex fuzzy relation of $W$ and $X, B$ a complex fuzzy relation of $X$ and $Y$, and $C$ a complex fuzzy relation of $Y$ and $Z$. Then, $A \circ(B \circ C)=(A \circ B) \circ C$.

Proposition 12 (see [15]). Let $X, Y$, and $Z$ be universes, $A$ and $B$ two complex fuzzy relations of $X$ and $Y$, and $C$ a complex fuzzy relation of $Y$ and $Z$. Then,

(i) $(A \cup B) \circ C=(A \circ C) \cup(B \circ C)$,

(ii) If $A \subseteq B$, then $A \circ C \subseteq B \circ C$.

Definition 13 (see [15]). Let $A$ and $B$ be two complex fuzzy sets on $X, \mu_{A}(x)=r_{A}(x) \cdot e^{i \arg _{A}(x)}$ and $\mu_{B}(x)=r_{B}(x) \cdot e^{i \arg _{B}(x)}$ their membership functions, respectively. We say that $A$ is greater than $B$, denoted by $A \supseteq B$ or $B \subseteq A$, if for any $x \in X, r_{A}(x) \leq$ $r_{B}(x)$, and $\arg _{A}(x) \leq \arg _{B}(x)$.

Definition 14 (see [11]). A complex Atanassov's intuitionistic fuzzy set $A$, defined on a universe of discourse $U$, is characterized by membership and nonmembership functions $\mu_{A}(x)$ and $\gamma_{A}(x)$, respectively, that assign to any element $x \in X$ a complex-valued grade of both membership and nonmembership functions in $A$.

By definition, the values of $\mu_{A}(x), \gamma_{A}(x)$, and their sum may receive all lying within the unit circle in the complex plane and are of the form $\mu_{A}(x)=r_{A}(x) \cdot e^{i \omega_{\mu_{A}}(x)}$ for membership function in $A$ and $\gamma_{A}(x)=k_{A}(x) \cdot e^{i \omega_{\gamma_{A}}(x)}$ for nonmembership function in $A$, where $i=\sqrt{-1}$, each of $r_{A}(x)$ and $k_{A}(x)$ is real-valued and both belong to the closed unit interval $[0,1]$ such that $0 \leq r_{A}(x)+k_{A}(x) \leq 1$; also, $\omega_{\mu_{\mathrm{A}}}(x)$ and $\omega_{\gamma_{A}}(x)$ are real-valued. We represent the CAIFS $A$ as

$$
A=\left\{\left\langle x, \mu_{A}(x), \gamma_{A}(x)\right\rangle: x \in X\right\},
$$

where $\mu_{A}(x): X \rightarrow\{a|a \in C| a \mid, \leq 1\}, \gamma_{A}(x): X \rightarrow\left\{a^{\prime} \mid\right.$ $\left.a^{\prime} \in C,\left|a^{\prime}\right| \leq 1\right\}$ and $\left|\mu_{A}(x)+\gamma_{A}(x)\right| \leq 1$.

Proposition 15 (see [11]). If $A$ is any CFS denoted by $A=$ $\left\{\left(x, \mu_{A}(x)\right): x \in X\right\}$, then we can represent the set $A$ as a set of CAIFS as follows:

$$
A=\left\{\left(x, \mu_{A}(x), \gamma_{A}(x)\right): x \in X\right\},
$$

where the nonmembership of a CAIFS A equals the complement of CFS $A$ (i.e., $\gamma_{A}(x)=\mu_{\bar{A}}(x)$ ).

Definition 16 (see [11]). Let $A=\left\{\left\langle x, \mu_{A}(x), \gamma_{A}(x)\right\rangle: x \in X\right\}$ be a complex Atanassov's intuitionistic fuzzy set. Define the complement of $A, c(A)$, as

$$
\begin{aligned}
c(A) & =\left\{\left\langle x, \gamma_{A}(x), \mu_{A}(x)\right\rangle: x \in X\right\} \\
& =\left\{\left\langle x, k_{A}(x) \cdot e^{i\left(\omega^{k} \bar{A}(x)\right)}, r_{A}(x) \cdot e^{i\left(\omega^{r} \bar{A}(x)\right)}\right\rangle: x \in X\right\},
\end{aligned}
$$

where $\omega_{\bar{A}}(x)=\omega_{A}(x), 2 \pi-\omega_{A}(x)$, or $\omega_{A}(x)+\pi$.
Definition 17 (see [11]). Let $A$ and $B$ be two CAIFSs on universe of discourse $X$, with complex-valued membership and nonmembership functions. The complex Atanassov's intuitionistic fuzzy union of $A$ and $B$, denoted by $A \cup B$, is specified by the following function:

$$
\begin{aligned}
E: & \left\{\left(a, a^{\prime}\right)\left|a, a^{\prime} \in C:\right| a+a^{\prime}|\leq 1,| a|\leq 1,| a^{\prime} \mid \leq 1\right\} \\
& \times\left\{\left(b, b^{\prime}\right)\left|b, b^{\prime} \in C:\right| b+b^{\prime}|\leq 1,| b|\leq 1,| b^{\prime} \mid \leq 1\right\} \\
& \longrightarrow\left\{\left(d, d^{\prime}\right)\left|d, d^{\prime} \in C:\right| d+d^{\prime}|\leq 1,| d|\leq 1,| d^{\prime} \mid \leq 1\right\},
\end{aligned}
$$

where $a, b$, and $d$ and $a^{\prime}, b^{\prime}$, and $d^{\prime}$ are the membership and nonmembership functions of $A, B$, and $A \cup B$, respectively. $E$ assigns a complex value,

$$
\begin{aligned}
& E\left(\left(a, a^{\prime}\right),\left(b, b^{\prime}\right)\right) \\
&=\left(E_{\mu}(a, b), E_{\gamma}\left(a^{\prime}, b^{\prime}\right)\right) \\
&=\left(\mu_{A \cup B}(x), \gamma_{A \cup B}(x)\right)=\left(d, d^{\prime}\right), \\
& \forall x \in X .
\end{aligned}
$$

The complex Atanassov's intuitionistic fuzzy union function, $E$, must satisfy at least the following axiomatic requirements, for any $a, b, c, d, a^{\prime}, b^{\prime}, c^{\prime}$, and $d^{\prime} \in\{x: x \in C,|x| \leq$ $1\}$.

(i) Axiom 1: $\left|E_{\mu}(a, 0)\right|=|a|$ and $\left|E_{\gamma}\left(a^{\prime}, 1\right)\right|=\left|a^{\prime}\right|$ (boundary condition).

(ii) Axiom 2: $E_{\mu}(a, b)=E_{\mu}(b, a)$ and $E_{\gamma}\left(a^{\prime}, b^{\prime}\right)=$ $E_{\gamma}\left(b^{\prime}, a^{\prime}\right)$ (commutative condition).

(iii) Axiom 3: If $|b| \leq|d|$, then $\left|E_{\mu}(a, b)\right| \leq\left|E_{\mu}(a, d)\right|$, and if $\left|b^{\prime}\right| \leq\left|d^{\prime}\right|$, then $\left|E_{\gamma}\left(a^{\prime}, b^{\prime}\right)\right| \leq\left|E_{\gamma}\left(a^{\prime}, d^{\prime}\right)\right|$ (monotonic condition).

(iv) Axiom 4: $E_{\mu}\left(E_{\mu}(a, b), c\right)=E_{\mu}\left(a, E_{\mu}(b, c)\right)$ and $E_{\gamma}\left(E_{\gamma}\left(a^{\prime}, b^{\prime}\right), c^{\prime}\right)=E_{\gamma}\left(a^{\prime}, E_{\gamma}\left(b^{\prime}, c^{\prime}\right)\right)$ (associative condition).

In some cases, it may be desirable that the following requirements are also satisfied.

(v) Axiom 5: $E$ is a continuous function (continuity).

(vi) Axiom 6: $1 \geq\left|E_{\mu}(a, a)\right| \geq|a|$ and $0 \leq\left|E_{\gamma}\left(a^{\prime}, a^{\prime}\right)\right| \leq$ $\left|a^{\prime}\right|$ (superidempotency).

(vii) Axiom 7: $|a| \leq|c|$ and $|b| \leq|d| \Rightarrow\left|E_{\mu}(a, b)\right| \leq$ $\left|E_{\mu}(c, d)\right|$. Also, $\left|a^{\prime}\right| \geq\left|c^{\prime}\right|$ and $\left|b^{\prime}\right| \geq\left|d^{\prime}\right| \Rightarrow$ $\left|E_{\gamma}\left(a^{\prime}, b^{\prime}\right)\right| \geq\left|E_{\gamma}\left(c^{\prime}, d^{\prime}\right)\right|$ (strict monotonicity).

We may represent the complex Atanassov's intuitionistic fuzzy union in the following manner:

$$
A \cup B=\left\{\left\langle x, \mu_{A \cup B}(x), \gamma_{A \cup B}(x)\right\rangle: x \in X\right\},
$$

where $\mu_{A \cup B}(x)=\left[r_{A}(x) S_{\mu} r_{B}(x)\right] \cdot e^{i \omega_{\mu A \cup B}(x)}$ and $\gamma_{A \cup B}(x)=$ $\left[k_{A}(x) S_{\gamma} k_{B}(x)\right] \cdot e^{i \omega_{\gamma A \cup B}(x)}$. 
While the phase term for membership and nonmembership functions belongs to $(0,2 \pi]$, we define $\omega_{\mu A \cup B}(x)=$ $\omega_{A \cup B}(x)=\omega_{\gamma A \cup B}(x)$ with some forms that Buckley [13] presented to calculate $\omega_{A \cup B}(x)$ as follows:

(i) Sum: $\omega_{A \cup B}(x)=\omega_{A}(x)+\omega_{B}(x)$.

(ii) $\operatorname{Max}: \omega_{A \cup B}(x)=\max \left(\omega_{A}(x), \omega_{B}(x)\right)$.

(iii) Min: $\omega_{A \cup B}(x)=\min \left(\omega_{A}(x), \omega_{B}(x)\right)$.

(iv) "Winner Takes All":

$$
\omega_{A \cup B}(x)= \begin{cases}\omega_{A}(x) & r_{A}>r_{B}, \\ \omega_{B}(x) & r_{A}<r_{B} .\end{cases}
$$

Definition 18 (see [11]). Let $A$ and $B$ be two CAIFSs on universe of discourse $X$, with complex-valued membership and nonmembership functions. The complex Atanassov's intuitionistic fuzzy intersection of $A$ and $B$, denoted by $A \cap B$, is specified by the following function:

$$
\begin{aligned}
Q & :\left\{\left(a, a^{\prime}\right)\left|a, a^{\prime} \in C:\right| a+a^{\prime}|\leq 1,| a|\leq 1,| a^{\prime} \mid \leq 1\right\} \\
& \times\left\{\left(b, b^{\prime}\right)\left|b, b^{\prime} \in C:\right| b+b^{\prime}|\leq 1,| b|\leq 1,| b^{\prime} \mid \leq 1\right\} \\
& \longrightarrow\left\{\left(d, d^{\prime}\right)\left|d, d^{\prime} \in C:\right| d+d^{\prime}|\leq 1,| d|\leq 1,| d^{\prime} \mid \leq 1\right\},
\end{aligned}
$$

where $a, b$, and $d$ and $a^{\prime}, b^{\prime}$, and $d^{\prime}$ are the membership and nonmembership functions of $A, B$, and $A \cap B$, respectively. $Q$ assigns a complex value,

$$
\begin{aligned}
Q\left(\left(a, a^{\prime}\right),\left(b, b^{\prime}\right)\right) \\
\quad=\left(Q_{\mu}(a, b), Q_{\gamma}\left(a^{\prime}, b^{\prime}\right)\right) \\
\quad=\left(\mu_{A \cap B}(x), \gamma_{A \cap B}(x)\right)=\left(d, d^{\prime}\right), \quad \forall x \in X .
\end{aligned}
$$

The complex Atanassov's intuitionistic fuzzy intersection function, $Q$, must satisfy at least the following axiomatic requirements, for any $a, b, c, d, a^{\prime}, b^{\prime}, c^{\prime}$, and $d^{\prime} \in\{x: x \in$ $C,|x| \leq 1\}$.

(i) Axiom 1: If $|b|=1$, then $\left|Q_{\mu}(a, b)\right|=|a|$, and if $\left|b^{\prime}\right|=$ 0 , then $\left|E_{\gamma}\left(a^{\prime}, b^{\prime}\right)\right|=\left|a^{\prime}\right|$ (boundary condition).

(ii) Axiom 2: $Q_{\mu}(a, b)=Q_{\mu}(b, a)$ and $Q_{\gamma}\left(a^{\prime}, b^{\prime}\right)=$ $Q_{\gamma}\left(b^{\prime}, a^{\prime}\right)$ (commutative condition).

(iii) Axiom 3: If $|b| \leq|d|$, then $\left|Q_{\mu}(a, b)\right| \leq\left|Q_{\mu}(a, d)\right|$, and if $\left|b^{\prime}\right| \leq\left|d^{\prime}\right|$, then $\left|Q_{\gamma}\left(a^{\prime}, b^{\prime}\right)\right| \leq\left|Q_{\gamma}\left(a^{\prime}, d^{\prime}\right)\right|$ (monotonic condition).

(iv) Axiom 4: $Q_{\mu}\left(Q_{\mu}(a, b), c\right)=Q_{\mu}\left(a, Q_{\mu}(b, c)\right)$ and $Q_{\gamma}\left(Q_{\gamma}\left(a^{\prime}, b^{\prime}\right), c^{\prime}\right)=Q_{\gamma}\left(a^{\prime}, Q_{\gamma}\left(b^{\prime}, c^{\prime}\right)\right)$ (associative condition).

In some cases, it may be desirable that the following requirements are satisfied:

(v) Axiom 5: $Q$ is a continuous function (continuity). (vi) Axiom 6: $0 \leq\left|Q_{\mu}(a, a)\right| \leq|a|$ and $1 \geq\left|Q_{\gamma}\left(a^{\prime}, a^{\prime}\right)\right| \geq$ $\left|a^{\prime}\right|$ (superidempotency).

(vii) Axiom 7: $|a| \leq|c|$ and $|b| \leq|d| \Rightarrow\left|Q_{\mu}(a, b)\right| \leq$ $\left|Q_{\mu}(c, d)\right|$. Also, $\left|a^{\prime}\right| \geq\left|c^{\prime}\right|$ and $\left|b^{\prime}\right| \geq\left|d^{\prime}\right| \Rightarrow$ $\left|Q_{\gamma}\left(a^{\prime}, b^{\prime}\right)\right| \geq\left|Q_{\gamma}\left(c^{\prime}, d^{\prime}\right)\right|$ (strict monotonicity).

We may represent the complex Atanassov's intuitionistic fuzzy intersection in the following manner:

$$
A \cap B=\left\{\left\langle x, \mu_{A \cap B}(x), \gamma_{A \cap B}(x)\right\rangle: x \in X\right\},
$$

where $\mu_{A \cap B}(x)=\left[r_{A}(x) T_{\mu} r_{B}(x)\right] \cdot e^{i \omega_{\mu A \cap B}(x)}, \gamma_{A \cap B}(x)=$ $\left[k_{A}(x) T_{\gamma} k_{B}(x)\right] \cdot e^{i \omega_{\gamma A \cap B}(x)}$.

We consider some forms to calculate the phase term, $\omega_{A \cap B}(x)$, such that the same possible choices are given to calculate the $\omega_{A \cup B}(x)$.

We can use any $S$-norm and $T$-norm that were introduced by Fathi [3] to demonstrate many examples on the amplitude terms on complex Atanassov's intuitionistic fuzzy union and intersection, respectively, with the membership and nonmembership functions of $A \cup B$ and $A \cap B$ are given, respectively, as follows:

For CAIF union,

$$
\begin{aligned}
& \mu_{A \cup B}(x)=\left[r_{A}(x) S_{\mu} r_{B}(x)\right] \cdot e^{i \omega_{\mu A \cup B}(x)}, \\
& \gamma_{A \cup B}(x)=\left[k_{A}(x) S_{\gamma} k_{B}(x)\right] \cdot e^{i \omega_{\gamma A \cup B}(x)} .
\end{aligned}
$$

For CAIF intersection,

$$
\begin{aligned}
& \mu_{A \cap B}(x)=\left[r_{A}(x) T_{\mu} r_{B}(x)\right] \cdot e^{i \omega_{\mu A \cap B}(x)}, \\
& \gamma_{A \cap B}(x)=\left[k_{A}(x) T_{\gamma} k_{B}(x)\right] \cdot e^{i \omega_{\gamma A \cap B}(x)} .
\end{aligned}
$$

Some examples of $S$-norm and $T$-norm are as follows.

(1) The standard $S$-norm:

for any two AIFSs $A=\left\{\left\langle x, \mu_{A}(x), \gamma_{A}(x)\right\rangle: x \in X\right\}$ and $B=\left\{\left\langle x, \mu_{B}(x), \gamma_{B}(x)\right\rangle: x \in X\right\}$ in a universe of discourse $X$, then $A \cup B$ is given by

$$
\begin{aligned}
A \cup B= & \left\{\left\langlex, \max \left(\mu_{A}(x), \mu_{B}(x)\right),\right.\right. \\
& \left.\left.\min \left(\gamma_{A}(x), \gamma_{B}(x)\right)\right\rangle: x \in X\right\} .
\end{aligned}
$$

This union is called the basic AIF union or Atanassov union, and it is the smallest AIFS containing both $A$ and $B$.

(2) Yager $S$-norm:

$S\left(\left(a, a^{\prime}\right),\left(b, b^{\prime}\right)\right)=S\left(S_{\omega}^{\mu}(a, b), S_{\omega}^{\gamma}\left(a^{\prime}, b^{\prime}\right)\right)$,

where $S_{\omega}^{\mu}(a, b)=\min \left(1,\left(a^{\omega}+b^{\omega}\right)^{1 / \omega}\right)$, and $S_{\omega}^{\gamma}\left(a^{\prime}\right.$, $\left.b^{\prime}\right)=1-\min \left(1,\left(\left(1-a^{\prime}\right)^{\omega}+\left(1-b^{\prime}\right)^{\omega}\right)^{1 / \omega}\right)$, with $\omega \in$ $(0, \infty)$. 
(3) The standard $T$-norm:

for any two AIFSs $A=\left\{\left\langle x, \mu_{A}(x), \gamma_{A}(x)\right\rangle: x \in X\right\}$ and $B=\left\{\left\langle x, \mu_{B}(x), \gamma_{B}(x)\right\rangle: x \in X\right\}$ in a universe of discourse $X$, then $A \cap B$ is given by

$$
\begin{aligned}
A \cap B= & \left\{\left\langlex, \min \left(\mu_{A}(x), \mu_{B}(x)\right),\right.\right. \\
& \left.\left.\max \left(\gamma_{A}(x), \gamma_{B}(x)\right)\right\rangle: x \in X\right\} .
\end{aligned}
$$

This intersection is called the basic AIF intersection or Atanassov intersection, and it is the largest AIFS contained in both $A$ and $B$.

(4) Yager $T$-norm:

$$
T\left(\left(a, a^{\prime}\right),\left(b, b^{\prime}\right)\right)=\left(T_{\omega}^{\mu}(a, b), T_{\omega}^{\gamma}\left(a^{\prime}, b^{\prime}\right)\right),
$$

where $T_{\omega}^{\mu}(a, b)=1-\min \left(1,\left((1-a)^{\omega}+(1-b)^{\omega}\right)^{1 / \omega}\right)$, and $T_{\omega}^{\gamma}\left(a^{\prime}, b^{\prime}\right)=\min \left(1,\left(\left(a^{\prime}\right)^{\omega}+\left(b^{\prime}\right)^{\omega}\right)^{1 / \omega}\right)$, with $\omega \in$ $(0, \infty)$.

Definition 19 (see [15]). Let $A$ and $B$ be two complex fuzzy sets on $X$ and $Y$, respectively, where $A=\left\{\left\langle x, \mu_{A}(x)=\right.\right.$ $\left.\left.r_{A}(x) e^{i \omega_{r_{A}}(x)}\right\rangle: x \in X\right\}$ and $B=\left\{\left\langle y, \mu_{B}(y)=r_{B}(y) e^{i \omega_{r_{B}}(y)}\right\rangle\right.$ : $y \in Y\}$. The complex fuzzy union of $A$ and $B$ in different universe of discourse, denoted by $A \oplus B$, is specified by

$$
A \oplus B\left\{\left\langle(x, y), \mu_{A \oplus B}(x, y)\right\rangle:(x, y) \in X \times Y\right\},
$$

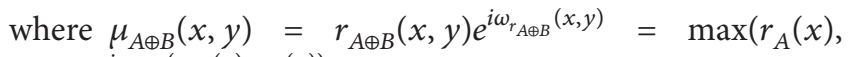
$\left.r_{B}(y)\right) e^{i \max \left(\omega_{r_{A}}(x), \omega_{r_{B}}(y)\right)}$.

Definition 20 (see [15]). Let $A$ and $B$ be two complex fuzzy sets on $X$ and $Y$, respectively, where $A=\left\{\left\langle x, \mu_{A}(x)=\right.\right.$ $\left.\left.r_{A}(x) e^{i \omega_{r_{A}}(x)}\right\rangle: x \in X\right\}$ and $B=\left\{\left\langle y, \mu_{B}(y)=r_{B}(y) e^{i \omega_{r_{B}}(y)}\right\rangle:\right.$ $y \in Y\}$. The complex fuzzy intersection of $A$ and $B$ in different universe of discourse, denoted by $A \otimes B$, is specified by

$$
A \otimes B\left\{\left\langle(x, y), \mu_{A \otimes B}(x, y)\right\rangle:(x, y) \in X \times Y\right\},
$$

where $\mu_{A \otimes B}(x, y)=r_{A \otimes B}(x, y) e^{i \omega_{r_{A \otimes B}}(x, y)}=\min \left(r_{A}(x)\right.$, $\left.r_{B}(y)\right) e^{i \min \left(\omega_{r_{A}}(x), \omega_{r_{B}}(y)\right)}$.

Definition 21 (see [15]). Let $A$ and $B$ be two complex fuzzy relations on $X \times Y$, where

$$
\begin{aligned}
A=\{ & \left\langle(x, y), \mu_{A}(x, y)=r_{A}(x, y) e^{i \omega_{r_{A}}(x, y)}\right\rangle: \\
& (x, y) \in X \times Y\}, \\
B=\{ & \left\langle(x, y), \mu_{B}(x, y)=r_{B}(x, y) e^{i \omega_{r_{B}}(x, y)}\right\rangle: \\
& (x, y) \in X \times Y\} .
\end{aligned}
$$

The complex fuzzy union relation of $A$ and $B$, denoted by $A \cup B$, is specified by

$$
A \cup B\left\{\left\langle(x, y), \mu_{A \cup B}(x, y)\right\rangle:(x, y) \in X \times Y\right\},
$$

where $\mu_{A \cup B}(x, y)=r_{A \cup B}(x, y) e^{i \omega_{r_{A \cup B}}(x, y)}=\max \left(r_{A}(x, y)\right.$, $\left.r_{B}(x, y)\right) e^{i \max \left(\omega_{r_{A}}(x, y), \omega_{r_{B}}(x, y)\right)}$.
Definition 22 (see [15]). Let $A$ and $B$ be two complex fuzzy relations on $X \times Y$, where

$$
\begin{aligned}
A= & \left\{\left\langle(x, y), \mu_{A}(x, y)=r_{A}(x, y) e^{i \omega_{r_{A}}(x, y)}\right\rangle:\right. \\
& (x, y) \in X \times Y\}, \\
B=\{ & \left\langle(x, y), \mu_{B}(x, y)=r_{B}(x, y) e^{i \omega_{r_{B}}(x, y)}\right\rangle: \\
& (x, y) \in X \times Y\} .
\end{aligned}
$$

The complex fuzzy intersection relation of $A$ and $B$, denoted by $A \cap B$, is specified by

$$
A \cap B\left\{\left\langle(x, y), \mu_{A \cap B}(x, y)\right\rangle:(x, y) \in X \times Y\right\},
$$

where $\mu_{A \cap B}(x, y)=r_{A \cap B}(x, y) e^{i \omega_{r_{A \cap B}}(x, y)}=\min \left(r_{A}(x, y)\right.$, $\left.r_{B}(x, y)\right) e^{i \min \left(\omega_{r_{A}}(x, y), \omega_{r_{B}}(x, y)\right)}$.

We call the relation $I$ an identical equal relation on $X$, if its membership is

$$
\mu_{I}(x, y)= \begin{cases}1 & \text { if } x=y \\ 0 & \text { if } x \neq y\end{cases}
$$

Definition 23 (see [15]). Let $A \in \mathrm{CFS}(U \times U)$, if $\mu_{A}(x, x)=$ 1 for all $x \in X$, then $A$ is a reflexive complex fuzzy relation.

Definition 24 (see [15]). Let $A \in \operatorname{CFS}(X \times Y)$, then $A^{T} \in$ CFS $(Y \times X)$ is a replacement of $A$, where

$$
\mu_{A^{T}}(y, x)=\mu_{A}(x, y) \quad \forall(x, y) \in X \times Y .
$$

Proposition 25 (see [15]). Let $A$ and $B$ be two complex fuzzy relations. Then, the following hold:

(1) $\left(A^{T}\right)^{T}=A$,

(2) $(A \cup B)^{T}=A^{T} \cup B^{T}$ and $(A \cap B)^{T}=A^{T} \cap B^{T}$,

(3) $(A \circ B)^{T}=B^{T} \circ A^{T}$ and $\left(A^{n}\right)^{T}=\left(A^{T}\right)^{n}$,

(4) $A \subseteq B$ if and only if $B^{T} \subseteq A^{T}$.

Definition 26 (see [15]). Let $A \in \mathrm{CFS}(X \times Y)$. Then $A$ is a symmetrical complex Atanassov's intuitionistic fuzzy relation if $\mu_{A}(x, y)=\mu_{A^{T}}(x, y)$ for all $(x, y) \in X \times Y$.

Definition 27 (see [15]). Let $A \in \mathrm{CFS}(X \times X)$. Then $A$ is a transitive complex fuzzy relation if $A \circ A \subseteq A$.

\section{Distance Measure on Complex Atanassov's Intuitionistic Fuzzy Sets}

In 1962, Hausdorff introduced the concept of distance as a measure of similarity or difference between sets [27]. Distance measure is very important because the "spatial distribution" of sets is fixed with respect to selected reference set [28]. Therefore, distance measure is used in significant real-life application, for instance, reasoning and decision making [28-40], fuzzy number ranking, and information retrieval in databases [41]. Also, in [28], Yang and Chiclana 
proved that the 3D distance of AIFS (i.e., the distance measured with three parameters membership, nonmembership, and hesitation functions) is useless and is not necessary because two-dimensional distance functions (membership and nonmembership functions) have already provided a simple and concise expression of the distance between two AIFSs. All distance measures in fuzzy set and intuitionistic fuzzy set are dealing with real-valued parameter, membership, and/or nonmembership functions, which only represented the uncertainty semantic. In the case of CAIFS, these parameters are complex-valued parameters and have two terms, uncertainty and periodicity semantics, where the uncertainty semantic is analogous to the one in traditional FS and/or AIFS. Therefore, the priority now is to introduce a distance measure that may involve both uncertainty and periodic semantics in one structure to confer the concepts of CFS and CAIFS a simple and concise expression. In application 1, we illustrate a kind of MADM problem with an additional factor, the periodic factors (i.e., MADM problems in CIF fields).

In this section, we generalise a distance measure on CAIFSs to implement a model which is utilized to select the best product with multiple attributes based on two dimensions. One of them is the periodic dimension which is considered as a critical influence to take a suitable decision. Song et al. [12] introduced a model to select a proper supplier based on distance measure of AIFS. They presented the definition of distance between two AIFSs. Here, we generalise their definition to CAIFSs and use this new approach of distance to find out the best periodic product from several selected products. This is a kind of MADM problem with an additional factor, the periodic factor (i.e., MADM problems in CIF fields).

Definition 28. If $A$ and $B$ are CAIFSs in a universe of discourse $X$, where $A=\left\{\left(x, r_{A}(x) e^{i \omega_{r_{A}}(x)}, k_{A}(x) e^{i \omega_{k_{A}}(x)}\right)\right\}$, and $B=\left\{\left(x, r_{B}(x) e^{i \omega_{r_{B}}(x)}, k_{B}(x) e^{i \omega_{k_{B}}(x)}\right)\right\}$, then

(1) $A \subset B$ if and only if $r_{A}(x)<r_{B}(x)$ and $k_{A}(x)>k_{B}(x)$, for amplitude terms and the phase terms (arguments) $\omega_{r_{A}}(x)<\omega_{r_{B}}(x)$ and $\omega_{k_{A}}(x)>\omega_{k_{B}}(x)$, for all $x \in X$.

(2) $A=B$ if and only if $r_{A}(x)=r_{B}(x)$ and $k_{A}(x)=k_{B}(x)$, for amplitude terms and the phase terms (arguments) $\omega_{r_{A}}(x)=\omega_{r_{B}}(x)$ and $\omega_{k_{A}}(x)=\omega_{k_{B}}(x)$, for all $x \in X$.

Let CIFS $(X)$ be the set of all complex Atanassov's intuitionistic fuzzy sets on $X$.

Definition 29. A distance of complex Atanassov's intuitionistic fuzzy sets is a function $d: \operatorname{CIFS}(X) \times \operatorname{CIFS}(X) \rightarrow[0,1]$ for any $A, B$, and $C \in \operatorname{CIFS}(X)$, satisfying the following properties:

(D1) $0 \leq d(A, B) \leq 1$.

(D2) $d(A, B)=0$ if and only if $A=B$.

(D3) $d(A, B)=d(B, A)$.

(D4) If $A \subset B \subset C$ and $A, B, C \in \operatorname{CIFS}(X)$, then $d(A, C)>d(A, B), d(A, C)>d(B, C)$.
We introduce a function $d: \operatorname{CIFS}(X) \times \operatorname{CIFS}(X) \rightarrow$ $[0,1]$ between CAIFSs $A$ and $B$, defined as follows:

$$
\begin{aligned}
& d(A, B) \\
& =\frac{1}{2 \sum_{i=1}^{n} w_{i}} \\
& \quad \times\left[\sum _ { i = 1 } ^ { n } w _ { i } \left[\left(\alpha_{1} \cdot\left|r_{A}\left(x_{i}\right)-r_{B}\left(x_{i}\right)\right|\right.\right.\right. \\
& +\beta_{1} \cdot\left|k_{A}\left(x_{i}\right)-k_{B}\left(x_{i}\right)\right| \\
& +\sigma_{1} \cdot \max \left(\left|r_{A}\left(x_{i}\right)-r_{B}\left(x_{i}\right)\right|\right. \\
& \left.\quad\left|k_{A}\left(x_{i}\right)-k_{B}\left(x_{i}\right)\right|\right) \\
& +\frac{1}{2 \pi}\left(\alpha_{2} \cdot\left|\omega_{r_{A}}\left(x_{i}\right)-\omega_{r_{B}}\left(x_{i}\right)\right|+\beta_{2}\right. \\
& \quad \cdot\left|\omega_{k_{A}}\left(x_{i}\right)-\omega_{k_{B}}\left(x_{i}\right)\right| \\
& +\sigma_{2} \cdot \max \left(\left|\omega_{r_{A}}\left(x_{i}\right)-\omega_{r_{B}}\left(x_{i}\right)\right|,\right. \\
&
\end{aligned}
$$

where $\alpha_{1}, \beta_{1}, \sigma_{1}, \alpha_{2}, \beta_{2}$, and $\sigma_{2} \in[0,1], \alpha_{1}+\beta_{1}+\sigma_{1}=1$ and $\alpha_{2}+\beta_{2}+\sigma_{2}=1 . w_{i} \in[0,1], i \in\{1,2, \ldots, m\}$.

Theorem 30. The function $d(A, B)$ defined in (34) is a distance measure of complex Atanassov's intuitionistic fuzzy set between two CAIFSs $A$ and $B$ in $X$.

Proof. (1) By definition of CAIFS, we have for all $x_{i}$ in $X$, where $i=1,2, \ldots, n$, each of $r_{A}\left(x_{i}\right), r_{B}\left(x_{i}\right)$, $k_{A}\left(x_{i}\right)$, and $r_{B}\left(x_{i}\right)$ lies in the interval $[0,1]$. Also each of $\omega_{r_{A}}\left(x_{i}\right), \omega_{r_{B}}\left(x_{i}\right), \omega_{k_{A}}\left(x_{i}\right)$, and $\omega_{k_{B}}\left(x_{i}\right)$ lies in the interval $[0,2 \pi]$. So it is easy to conclude that each of the following lies between 0 and $1:\left|r_{A}\left(x_{i}\right)-r_{B}\left(x_{i}\right)\right|$, and $\left|k_{A}\left(x_{i}\right)-k_{B}\left(x_{i}\right)\right|$. Also, $\left|\omega_{r_{A}}\left(x_{i}\right)-\omega_{r_{B}}\left(x_{i}\right)\right|$ and $\left|\omega_{k_{A}}\left(x_{i}\right)-\omega_{k_{B}}\left(x_{i}\right)\right|$ lie between 0 and $2 \pi$.

So we have

$$
\begin{aligned}
& d(A, B) \\
& \leq \frac{1}{2 \sum_{i=1}^{n} w_{i}} \\
& \quad \times\left[\sum _ { i = 1 } ^ { n } w _ { i } \left[\left(\alpha_{1} \cdot 1+\beta_{1} \cdot 1+\sigma_{1} \cdot \max (1,1)\right)\right.\right. \\
& \left.\quad+\frac{1}{2 \pi}\left(\alpha_{2} \cdot 2 \pi+\beta_{2} \cdot 2 \pi+\sigma_{2} \cdot \max (2 \pi, 2 \pi)\right]\right] \\
& =\frac{1}{2 \sum_{i=1}^{n} w_{i}}\left[\sum_{i=1}^{n} w_{i}\left[\left(\alpha_{1}+\beta_{1}+\sigma_{1}\right)+\left(\alpha_{2}+\beta_{2}+\sigma_{2}\right)\right]\right]
\end{aligned}
$$


Since $\alpha_{1}+\beta_{1}+\sigma_{1}=1$ and $\alpha_{2}+\beta_{2}+\sigma_{2}=1$, we have

$$
0 \leq d(A, B) \leq \frac{1}{2 \sum_{i=1}^{n} w_{i}}\left[\sum_{i=1}^{n} 2 w_{i}\right]=1 .
$$

(2) By Definition 28, it is easy to see that $d(A, B)$ satisfies the second and third conditions of Definition 29.

(3) By using Definition 28,

$$
\begin{gathered}
1 \geq k_{A}\left(x_{i}\right) \geq k_{B}\left(x_{i}\right) \geq k_{C}(x) \geq 0, \\
2 \pi \geq \omega_{k_{A}}\left(x_{i}\right) \geq \omega_{k_{B}}\left(x_{i}\right) \geq \omega_{k_{C}}\left(x_{i}\right) \geq 0 .
\end{gathered}
$$

Then, we can conclude that

$$
\begin{aligned}
\left|r_{A}\left(x_{i}\right)-r_{B}\left(x_{i}\right)\right| & \leq\left|r_{A}\left(x_{i}\right)-r_{C}\left(x_{i}\right)\right|, \\
\left|\omega_{r_{A}}\left(x_{i}\right)-\omega_{r_{B}}\left(x_{i}\right)\right| & \leq\left|\omega_{r_{A}}\left(x_{i}\right)-\omega_{r_{C}}\left(x_{i}\right)\right|, \\
\left|k_{A}\left(x_{i}\right)-k_{B}\left(x_{i}\right)\right| & \geq\left|k_{A}\left(x_{i}\right)-k_{C}\left(x_{i}\right)\right|, \\
\left|\omega_{k_{A}}\left(x_{i}\right)-\omega_{k_{B}}\left(x_{i}\right)\right| & \geq\left|\omega_{k_{A}}\left(x_{i}\right)-\omega_{k_{C}}\left(x_{i}\right)\right| .
\end{aligned}
$$

So, $d(A, B) \leq d(A, C)$.

Analogously, we can get $d(B, C) \leq d(A, C)$.

From (82), (83), and (84), we conclude that $d(A, B)$ is a distance measure between CAIFSs $A$ and $B$.

In the following example, Application 31, we apply a selection model from [12]. Also, we generalise the selection operation to be covered by CAIFS to get the desired car or the best alternative for a company to select a car from four models of cars with its production date simultaneously. So we have a two-dimensional problem, namely, model of cars and production date of the cars.

Application 31. Suppose Company $X$ decides to purchase cars from a carmaker $Y$. The carmaker $Y$ offers Company $X$ some information on four models of cars with different production dates for each model. So Company $X$ has four models $\left(\mathrm{Car}_{1}\right.$, $\mathrm{Car}_{2}, \mathrm{Car}_{3}$, and $\mathrm{Car}_{4}$ ) to select with its production date simultaneously. The company's team of analysts agreed that five attributes should be considered. They are $A_{1}$ : reliability, $A_{2}$ : maximum payload, $A_{3}$ : purchasing cost, $A_{4}$ : maximum speed, and $A_{5}$ : durability. But these attributes will be affected and changed if the production date is different for the same model of cars. The decision made by the team will also depend on their knowledge and experience. So, we may get "yes," "no," "I do not know," or "I am not sure" as an answer to the selection of the desired cars. Consequently, as illustrated above, the best way to represent this sort of information is by using CAIFS (i.e., represent the information on people's decision which happens periodically), in which with each new product model of car, the consumers have different opinions and mentalities. To be more explicit, assume that the company's team of analysts has suggested an ideal car, before getting the characteristic information from carmaker $Y$. The target of the team is to select a suitable car listed by carmaker $Y$ that is most likely to be the ideal car. Then, every analyst in the selection team gives each car's attribute a score 1 or -1 to indicate whether the car is suitable for the attribute or not and gives a score 0 when he is not sure of the model and production date of car.
For instance, suppose the team of analysts feels that at least $70 \%$ of them believe that the ideal model car is suitable at the first attribute; and not more than $10 \%$ of the analysts believe that the ideal model car is poor at the first attribute, in which this process is utilized to calculate the amplitude terms for both membership and nonmembership functions respectively, in CAIFS. The phase terms that present production date for first attribute of ideal car can be given as follows: if the team of analysts thought that at least $80 \%$ of them believe that the ideal production date of car is suitable at the first attribute; and not more than $15 \%$ of them believe that the ideal production date of car is poor. So the ideal car's first attribute can be presented as $\left(0.7 e^{i 2 \pi(0.8)}, 0.1 e^{i 2 \pi(0.15)}\right)$. In this way, all data can be obtained in the form of CAIFS, where both amplitude and phase terms can represent information of Atanassov's intuitionistic uncertainty (see Table 1).

To continue our example, we recall a proper model to get a suitable car that is most likely to be the ideal car (see [12]).

Let $d\left(\mathrm{Car}_{j}\right.$, I.C.) be a distance measure between complex Atanassov's intuitionistic fuzzy sets $\mathrm{Car}_{j}$ and I.C. So, the model can be introduced as follows:

$$
\begin{gathered}
M=\left\{\operatorname{Car}_{j} \mid d\left(\operatorname{Car}_{j}, \text { I.C. }\right)\right. \\
=\min \left(\sum_{i=1}^{n} d\left(\operatorname{Car}_{1, i}, \text { I.C }_{i} .\right), \ldots,\right. \\
\left.\sum_{i=1}^{n} d\left(\operatorname{Car}_{k, i}, \text { I.C }_{i} \cdot\right)\right), \\
j=1, \ldots, k, i=1, \ldots, n\} .
\end{gathered}
$$

Suppose the team of analysts gives the weight for each attribute as follows.

Let $w_{1}=0.2, w_{2}=0.1, w_{3}=0.5, w_{4}=0.7$, and $w_{5}=$ 0.5 present the weight for each attribute. Let $\alpha_{1}=0.2, \beta_{1}=$ 0.5 , and $\sigma_{1}=0.3$ be the weight for the amplitude term, while $\alpha_{2}=0.4, \beta_{2}=0.4$, and $\sigma_{2}=0.2$ be the weight for the phase term.

To identify the ideal car from the information given in Table 1, we substitute the data into distance formula (Definition 29) to evaluate the values between each car $\left(\mathrm{Car}_{j}, j=1,2,3,4\right)$ and the ideal car (I.C.):

$$
\begin{aligned}
& d\left(\operatorname{Car}_{1}, \text { I.C. }\right) \\
& =\sum_{i=1}^{5} d\left(\operatorname{Car}_{1, i}, \text { I.C }_{i} .\right) \\
& =\frac{1}{2 \cdot(2.9)} \\
& \quad \times\left[\sum _ { i = 1 } ^ { 5 } w _ { i } \left[\left(\alpha_{1} \cdot\left|r_{A}\left(x_{i}\right)-r_{B}\left(x_{i}\right)\right|\right.\right.\right. \\
& \quad+\beta_{1} \cdot\left|k_{A}\left(x_{i}\right)-k_{B}\left(x_{i}\right)\right| \\
& \quad+\sigma_{1} \cdot \max \left(\left|r_{A}\left(x_{i}\right)-r_{B}\left(x_{i}\right)\right|,\right. \\
& \left.\left.\quad\left|k_{A}\left(x_{i}\right)-k_{B}\left(x_{i}\right)\right|\right)\right)
\end{aligned}
$$


TABLE 1: Car data and the ideal car.

\begin{tabular}{cccccc}
\hline & $\mathrm{Car}_{1}$ & $\mathrm{Car}_{2}$ & $\mathrm{Car}_{3}$ & $\mathrm{Car}_{4}$ & Ideal Car $(\mathrm{I} . \mathrm{C})$. \\
\hline$A_{1}$ & $\left(0.4 e^{i 2 \pi(0.3)}, 0.3 e^{i 2 \pi(0.2)}\right)$ & $\left(0.7 e^{i 2 \pi(0.6)}, 0.3 e^{i 2 \pi(0.3)}\right)$ & $\left(0.3 e^{i 2 \pi(0.4)}, 0.6 e^{i 2 \pi(0.4)}\right)$ & $\left(0.2 e^{i 2 \pi(0.8)}, 0.5 e^{i 2 \pi(0.1)}\right)$ & $\left(0.7 e^{i 2 \pi(0.5)}, 0.1 e^{i 2 \pi(0.3)}\right)$ \\
$A_{2}$ & $\left(0.7 e^{i 2 \pi(0.5)}, 0.1 e^{i 2 \pi(0.4)}\right)$ & $\left(0.4 e^{i 2 \pi(0.9)}, 0.2 e^{i 2 \pi(0.1)}\right)$ & $\left(0.6 e^{i 2 \pi(0.6)}, 0.3 e^{i 2 \pi(0.4)}\right)$ & $\left(0.7 e^{i 2 \pi(0.3)}, 0.3 e^{i 2 \pi(0.3)}\right)$ & $\left(0.4 e^{i 2 \pi(0.6)}, 0.5 e^{i 2 \pi(0.2)}\right)$ \\
$A_{3}$ & $\left(0.5 e^{i 2 \pi(0.6)}, 0.3 e^{i 2 \pi(0.2)}\right)$ & $\left(0.7 e^{i 2 \pi(0.7)}, 0.2 e^{i 2 \pi(0.3)}\right)$ & $\left(0.3 e^{i 2 \pi(0.4)}, 0.5 e^{i 2 \pi(0.6)}\right)$ & $\left(0.6 e^{i 2 \pi(0.5)}, 0.1 e^{i 2 \pi(0.3)}\right)$ & $\left(0.5 e^{i 2 \pi(0.5)}, 0.3 e^{i 2 \pi(0.1)}\right)$ \\
$A_{4}$ & $\left(0.7 e^{i 2 \pi(0.7)}, 0.1 e^{i 2 \pi(0.2)}\right)$ & $\left(0.4 e^{i 2 \pi(0.6)}, 0.3 e^{i 2 \pi(0.1)}\right)$ & $\left(0.7 e^{i 2 \pi(0.7)}, 0.1 e^{i 2 \pi(0.1)}\right)$ & $\left(0.6 e^{i 2 \pi(0.5)}, 0.3 e^{i 2 \pi(0.4)}\right)$ & $\left(0.8 e^{i 2 \pi(0.7)}, 0.2 e^{i 2 \pi(0.1)}\right)$ \\
$A_{5}$ & $\left(0.4 e^{i 2 \pi(0.4)}, 0.2 e^{i 2 \pi(0.5)}\right)$ & $\left(0.5 e^{i 2 \pi(0.3)}, 0.3 e^{i 2 \pi(0.6)}\right)$ & $\left(0.7 e^{i 2 \pi(0.8)}, 0.2 e^{i 2 \pi(0.1)}\right)$ & $\left(0.4 e^{i 2 \pi(0.8)}, 0.4 e^{i 2 \pi(0.1)}\right)$ & $\left(0.6 e^{i 2 \pi(0.9)}, 0.1 e^{i 2 \pi(0.1)}\right)$ \\
\hline
\end{tabular}

$$
\begin{aligned}
&+\frac{1}{2 \pi}\left(\alpha_{2} \cdot\left|\omega_{r_{A}}\left(x_{i}\right)-\omega_{r_{B}}\left(x_{i}\right)\right|\right. \\
&+\beta_{2} \cdot\left|\omega_{k_{A}}\left(x_{i}\right)-\omega_{k_{B}}\left(x_{i}\right)\right| \\
&+\sigma_{2} \cdot \max \left(\left|\omega_{r_{A}}\left(x_{i}\right)-\omega_{r_{B}}\left(x_{i}\right)\right|,\right. \\
&\left.\left.\left.\left.\left|\omega_{k_{A}}\left(x_{i}\right)-\omega_{k_{B}}\left(x_{i}\right)\right|\right)\right)\right]\right] .
\end{aligned}
$$

For the first attribute $A_{1},(i=1)$, the distance is given as follows:

$$
\begin{aligned}
& d\left(\operatorname{Car}_{1,1}, \mathrm{I}_{1} \cdot\right) \\
& \begin{aligned}
&=\frac{1}{2 \cdot(2.9)}[0.2[(0.2 \cdot|0.4-0.7|+0.5 \cdot|0.3-0.1| \\
&+0.3 \cdot \max (0.3,0.2)) \\
&+ \frac{1}{2 \pi}(0.4 \cdot|0.3(2 \pi)-0.5(2 \pi)| \\
&+0.4 \cdot|0.2(2 \pi)-0.3(2 \pi)| \\
&+0.2 \cdot \max (0.2(2 \pi), 0.1(2 \pi)))]] \\
&=\frac{1}{2 \cdot(2.9)}[0.082] .
\end{aligned}
\end{aligned}
$$

Analogously, we calculate the distances for $\mathrm{Car}_{1}$ for each attribute,

$$
\begin{aligned}
d & \left(\mathrm{Car}_{1,2}, \mathrm{I}_{\mathrm{C}} \cdot\right) \\
& =\frac{1}{2 \cdot(2.9)}[0.054], d\left(\mathrm{Car}_{1,3}, \mathrm{I}_{\mathrm{C}} .\right) \\
& =\frac{1}{2 \cdot(2.9)}[0.05], d\left(\mathrm{Car}_{1,4},{\mathrm{I} . \mathrm{C}_{4} .}\right) \\
& =\frac{1}{2 \cdot(2.9)}[0.112], d\left(\mathrm{Car}_{1,5}, \mathrm{I}_{\mathrm{C}} .\right) \\
& =\frac{1}{2 \cdot(2.9)}[0.305] .
\end{aligned}
$$

Then,

$$
\begin{aligned}
d\left(\mathrm{Car}_{1}, \text { I.C. }\right)= & \frac{1}{2 \cdot(2.9)} \\
& \times[0.082+0.054+0.05+0.112+0.305] \\
= & 0.104 .
\end{aligned}
$$

Analogously, we calculate the distance values for $d\left(\mathrm{Car}_{2}\right.$, I.C. $)=0.842, d\left(\mathrm{Car}_{3}\right.$, I.C. $)=0.538$, and $d\left(\mathrm{Car}_{4}\right.$, I.C. $)=0.796$. Therefore, $d\left(\mathrm{Car}_{1}\right.$, I.C. $)=0.104$ is the smallest value, so $\mathrm{Car}_{1}$ is the desired car.

Note 1. Different weights of the criteria lead to different results. A company needs to set proper weights for the criteria, in order to get the proper result.

If we have the same model of cars but with different production date for the first attribute, surely the team of analysts will give us different values from those mentioned in the table above for the first attribute, because every year, the carmakers produce the same model of cars but with a slight improvement and difference. Even though the changes are slightly improved in the new models of cars, people's acceptance and appreciation may get changed to buy the new production model.

\section{Complex Atanassov's Intuitionistic Fuzzy Relation}

Fuzzy logics and systems open immense applications in engineering, medical, physics, automobiles, defense and security, internet and computer security, and other fields. It is a meaningful topic to introduce the concept of CAIF logics and systems which give us the ability to extend the realm of reallife applications in the CAIF environment. So, we develop the concept of complex Atanassov's intuitionistic fuzzy relations and compositions before introducing the concept of CAIF logics and systems.

The relation $R$ between the sets $X_{1}, \ldots, X_{n}$ is a subset of the Cartesian product $X_{1} \times \cdots \times X_{n}$. If we denote the relation above by $R\left(X_{1}, \ldots, X_{n}\right)$, then

$$
R\left(X_{1}, \ldots, X_{n}\right) \subset X_{1} \times \cdots \times X_{n} .
$$

Firstly, we should introduce the Cartesian product between complex Atanassov's intuitionistic fuzzy sets. In general, the Cartesian product between several CAIFSs is given as follows. 
Definition 32. Let $A_{1}, \ldots, A_{n}$ be complex Atanassov's intuitionistic fuzzy sets in $X_{1}, \ldots, X_{n}$, respectively. The Cartesian product $A_{1} \times \cdots \times A_{n}$ is a complex intuitionistic fuzzy set defined by

$$
\begin{aligned}
A_{1} \times \cdots \times A_{n} & \\
=\{ & \left\langle\left(x_{1}, \ldots, x_{n}\right), \mu_{A_{1} \times \cdots \times A_{n}}\left(x_{1}, \ldots, x_{n}\right),\right. \\
& \left.\gamma_{A_{1} \times \cdots \times A_{n}}\left(x_{1}, \ldots, x_{n}\right)\right\rangle: \\
& \left.\left(x_{1}, \ldots, x_{n}\right) \in X_{1} \times \cdots \times X_{n}\right\}
\end{aligned}
$$

where

$$
\begin{aligned}
& \mu_{A_{1} \times \cdots \times A_{n}}\left(x_{1}, \ldots, x_{n}\right) \\
&= \min \left(\mu_{A_{1}}\left(x_{1}\right), \ldots, \mu_{A_{n}}\left(x_{n}\right)\right) \\
&\left.\cdot e^{i \min \left(\omega_{\mu_{A_{1}}}\left(x_{1}\right), \ldots, \omega_{\mu_{A_{n}}}\left(x_{n}\right)\right)}\right], \\
& \gamma_{A_{1} \times \cdots \times A_{n}}\left(x_{1}, \ldots, x_{n}\right) \\
&=\left[\max \left(\gamma_{A_{1}}\left(x_{1}\right), \ldots, \gamma_{A_{n}}\left(x_{n}\right)\right)\right. \\
&\left.\quad \cdot e^{i \max \left(\omega_{\gamma_{A_{1}}}\left(x_{1}\right), \ldots, \omega_{\gamma_{A_{n}}}\left(x_{n}\right)\right)}\right] .
\end{aligned}
$$

In the case of two complex Atanassov's intuitionistic fuzzy sets $A$ and $B$ in $X$ and $Y$, respectively, we have

$$
\begin{aligned}
A & \times B \\
& =\left\{\left\langle(x, y), \mu_{A \times B}(x, y), \gamma_{A \times B}(x, y)\right\rangle:(x, y) \in X \times Y\right\},
\end{aligned}
$$

where

$$
\begin{aligned}
& \mu_{A \times B}(x, y)=\min \left(\mu_{A}(x), \mu_{B}(y)\right) \cdot e^{i \min \left(\omega_{\mu_{A}}(x), \omega_{\mu_{B}}(y)\right)}, \\
& \gamma_{A \times B}(x, y)=\max \left(\gamma_{A}(x), \gamma_{B}(y)\right) \cdot e^{i \max \left(\omega_{\gamma_{A}}(x), \omega_{\gamma_{B}}(y)\right)} .
\end{aligned}
$$

A special case is obtained when we generate a relation between two sets $X$ and $Y$. So, we present a definition of a complex Atanassov's intuitionistic fuzzy relation as follows.

Definition 33. A complex Atanassov's intuitionistic fuzzy relation $R(X, Y)$ between CAIFSs $U$ and $V$ is a subset of the product space $X \times Y$, where the complex membership and nonmembership functions of the relation $R(X, Y)$ are characterized by $\mu_{R}(x, y)$ and $\gamma_{R}(x, y)$, respectively, that assign each pair to each $(x, y) \in X \times Y$ a complex-valued grade of membership and nonmembership functions to the set $R(X, Y)$, where $x \in X$ and $y \in Y$. We write the relation $R(X, Y)$ as

$$
R(X, Y)=\left\{\left\langle(x, y), \mu_{R}(x, y), \gamma_{R}(x, y)\right\rangle \mid(x, y) \in X \times Y\right\} .
$$

The values $\mu_{R}(x, y)$ and $\gamma_{R}(x, y)$ may receive lie within the unit circle in the complex plane and are of the following form: $r(x) \cdot e^{i \omega_{\mu}(x)}$, and $k(x) \cdot e^{i \omega_{\gamma}(x)}(i=\sqrt{-1})$, where $r(x), k(x), \omega_{\mu}(x)$, and $\omega_{\gamma}(x)$ are all real-valued, with $r(x), k(x) \in[0,1]$, and $0 \leq r(x)+k(x) \leq 1$.

The membership and nonmembership functions in a CAISR represent both the degree of presence and absence of association, interaction, or interconnectedness, and the phase of association, interaction, or interconnectedness between the elements of two or more sets. Under the assertion that any of the complex fuzzy sets can be represented as a CAIFS (see [11, Proposition 2.3]), we rewrite Example 5 of Ramot et al. [4, page 183] in terms of CAIFS to demonstrate the utilization of CAIFR in real life.

Example 34. Let $X$ be the set of financial indicators or indexes of the American economy. Possible elements of this set are unemployment rate, inflation, interest rates, growth rate, GDP, Dow-Jones industrial average, and so forth. Let $Y$ be the set of financial indicators of the Japanese economy. Let the complex fuzzy relation $R(X, Y)$ represent the relation of influence of American financial indexes on Japanese financial indexes: " $y$ is influenced by $x$," where $x \in X$ and $y \in Y$.

The membership and nonmembership functions for the relation $R(X, Y), \mu(X, Y)$, and $\gamma(X, Y)$ are complex-valued, with an amplitude term and a phase term. The amplitude terms of membership and nonmembership functions indicate the degree of influence and noinfluence of an American financial index on a Japanese financial index, respectively. An amplitude term with a value close to one implies a large degree of influence, while a value close to zero suggests small to no influence. The phase terms of membership and nonmembership functions indicate the "phase" of influence and no-influence, or time lag that characterizes the influence and no influence of an American index on a Japanese index. Thus, the phase terms represent the time that elapses before the influence and no-influence of a certain occurrence in an American financial indicator is evident in a Japanese counterpart.

Consider for example, $\mu_{R}$ (Growth Rate, Export) and $\gamma_{R}$ (Growth Rate, Export), that is, the grade of membership and nonmembership associated with the statement "American growth rate influences and noinfluences Japanese export, respectively." The values of $\mu_{R}$ and $\gamma_{R}$ may be calculated from available economic statistics using a variety of methods (genetic algorithms, neural nets, etc.) or obtained from an expert. In this example, the latter of the two options is considered.

Suppose an expert was to state that "the influence of American growth rate on Japanese export is large, and the effect of a decline or increase in American growth is evident in Japanese export in three-five months." If $R(X, Y)$ was a traditional Atanassov's intuitionistic fuzzy relation, values of about 0.8 and 0.1 would be selected for the grade of membership and nonmembership, respectively, and all information regarding the time frame of the interaction between these two economic parameters would be lost. However, $R(X, Y)$ is a complex Atanassov's intuitionistic fuzzy relation, $\mu_{R}$ (Growth Rate, Export) and $\gamma_{R}$ (Growth Rate, Export) thus can be assigned a complex value which incorporates all of the information provided by the expert. 
Assume $R(X, Y)$ measures interactions between American and Japanese financial indicators in the limited time frame of 12 months. The following value may, therefore, be attributed to $\mu_{R}$ (Growth Rate, Export) and $\gamma_{R}$ (Growth Rate, Export):

$$
\begin{aligned}
& \mu_{R}(\text { Growth Rate, Export })=0.8 \cdot e^{(4 / 12) 2 \pi i}, \\
& \gamma_{R}(\text { Growth Rate, Export })=0.1 \cdot e^{(4 / 12) 2 \pi i} .
\end{aligned}
$$

Note that the amplitude terms selected are 0.8 and 0.1 , similar to the grade of membership and nonmembership of a traditional Atanassov's intuitionistic fuzzy set. The phase term is chosen to be 4 as an average of "three-five months," normalized by 12 months - the maximum timeframe the relation is designed to take into account for both membership phase and nonmembership phase functions.

Definition 35. Let $A$ and $B$ be two complex Atanassov's intuitionistic fuzzy sets on $X$ and $Y$, respectively, where

$$
\begin{gathered}
A=\left\{\left\langlex, \mu_{A}(x)=r_{A}(x) e^{i \omega_{r_{A}}(x)},\right.\right. \\
\left.\left.\gamma_{A}(x)=k_{A}(x) e^{i \omega_{k_{A}}(x)}\right\rangle: x \in X\right\}, \\
B=\left\{\left\langley, \mu_{B}(y)=r_{B}(y) e^{i \omega_{r_{B}}(y)},\right.\right. \\
\left.\left.\gamma_{B}(y)=k_{B}(y) e^{i \omega_{k_{B}}(y)}\right\rangle: y \in Y\right\} .
\end{gathered}
$$

The complex Atanassov's intuitionistic fuzzy union of $A$ and $B$ in different universe of discourse, denoted by $A \oplus B$, is specified by

$$
A \oplus B\left\{\left\langle(x, y), \mu_{A \oplus B}(x, y), \gamma_{A \oplus B}(x, y)\right\rangle:(x, y) \in X \times Y\right\},
$$

where $\mu_{A \oplus B}(x, y)=r_{A \oplus B}(x, y) e^{i \omega_{r_{A \oplus B}}(x, y)}=$ $\max \left(r_{A}(x), r_{B}(y)\right) e^{i \max \left(\omega_{r_{A}}(x), \omega_{r_{B}}(y)\right)}$ and $\gamma_{A \oplus B}(x, y)=$ $k_{A \oplus B}(x, y) e^{i \omega_{k_{A \oplus B}}(x, y)}=\min \left(k_{A}(x), k_{B}(y)\right) e^{i \min \left(\omega_{k_{A}}(x), \omega_{k_{B}}(y)\right)}$.

Example 36. Let

$$
\begin{aligned}
A= & \left\{\frac{\left(0.5 e^{i 1.3 \pi}, 0.4 e^{i 0.5 \pi}\right)}{-1}, \frac{\left(1.0 e^{i 1.5 \pi}, 0.0 e^{i 0.5 \pi}\right)}{0},\right. \\
& \left.\frac{\left(0.7 e^{i 0.3 \pi}, 0.2 e^{i 1.5 \pi}\right)}{1}\right\}, \\
B= & \left\{\frac{\left(0.8 e^{i 1.1 \pi}, 0.2 e^{i 0.7 \pi}\right)}{-1}, \frac{\left(0.4 e^{i 0.9 \pi}, 0.4 e^{i \pi}\right)}{0},\right. \\
& \left.\frac{\left(0.7 e^{i 0.9 \pi}, 0.2 e^{i 0.4 \pi}\right)}{1}\right\},
\end{aligned}
$$

then

$$
\begin{aligned}
A \oplus B= & \left\{\frac{\left(0.8 e^{i 1.3 \pi}, 0.2 e^{i 0.5 \pi}\right)}{(-1,-1)}, \frac{\left(0.5 e^{i 1.3 \pi}, 0.4 e^{i 0.5 \pi}\right)}{(-1,0)},\right. \\
& \frac{\left(0.7 e^{i 1.3 \pi}, 0.2 e^{i 0.4 \pi}\right)}{(-1,1)}, \frac{\left(1.0 e^{i 1.5 \pi}, 0.0 e^{i 0.5 \pi}\right)}{(0,-1)}, \\
& \frac{\left(1.0 e^{i 1.5 \pi}, 0.0 e^{i 0.5 \pi}\right)}{(0,0)}, \frac{\left(0.7 e^{i 0.9 \pi}, 0.2 e^{i 1.0 \pi}\right)}{(1,0)}, \\
& \frac{\left(0.8 e^{i 1.1 \pi}, 0.2 e^{i 0.7 \pi}\right)}{(1,-1)}, \frac{\left(0.7 e^{i 0.9 \pi}, 0.2 e^{i 1.0 \pi}\right)}{(1,0)}, \\
& \left.\frac{\left(0.7 e^{i 0.9 \pi}, 0.2 e^{i 0.4 \pi}\right)}{(1,1)}\right\} .
\end{aligned}
$$

Definition 37. Let $A$ and $B$ be two complex Atanassov's intuitionistic fuzzy sets on $X$ and $Y$, respectively, where

$$
\begin{gathered}
A=\left\{\left\langlex, \mu_{A}(x)=r_{A}(x) e^{i \omega_{r_{A}}(x)},\right.\right. \\
\left.\left.\gamma_{A}(x)=k_{A}(x) e^{i \omega_{k_{A}}(x)}\right\rangle: x \in X\right\}, \\
B=\left\{\left\langley, \mu_{B}(y)=r_{B}(y) e^{i \omega_{r_{B}}(y)},\right.\right. \\
\left.\left.\gamma_{B}(y)=k_{B}(y) e^{i \omega_{k_{B}}(y)}\right\rangle: y \in Y\right\} .
\end{gathered}
$$

The complex Atanassov's intuitionistic fuzzy intersection of $A$ and $B$ in different universe of discourse, denoted by $A \otimes B$, is specified by

$$
A \otimes B\left\{\left\langle(x, y), \mu_{A \otimes B}(x, y), \gamma_{A \otimes B}(x, y)\right\rangle:(x, y) \in X \times Y\right\},
$$

where $\mu_{A \otimes B}(x, y)=r_{A \otimes B}(x, y) e^{i \omega_{r_{A \otimes B}}(x, y)}=$ $\min \left(r_{A}(x), r_{B}(y)\right) e^{i \min \left(\omega_{r_{A}}(x), \omega_{r_{B}}(y)\right)}$ and $\gamma_{A \otimes B}(x, y)=$ $k_{A \otimes B}(x, y) e^{i \omega_{k_{A \otimes B}}(x, y)}=\max \left(k_{A}(x), k_{B}(y)\right) e^{i \max \left(\omega_{k_{A}}(x), \omega_{k_{B}}(y)\right)}$.

Example 38. Using the CAIFS $A$ and $B$ as in Example 36, we have

$$
\begin{aligned}
A \otimes B= & \left\{\frac{\left(0.5 e^{i 1.1 \pi}, 0.4 e^{i 0.7 \pi}\right)}{(-1,-1)}, \frac{\left(0.4 e^{i 0.9 \pi}, 0.4 e^{i 1.0 \pi}\right)}{(-1,0)},\right. \\
& \frac{\left(0.5 e^{i 0.9 \pi}, 0.4 e^{i 0.5 \pi}\right)}{(-1,1)}, \frac{\left(0.8 e^{i 1.1 \pi}, 0.2 e^{i 0.7 \pi}\right)}{(0,-1)}, \\
& \frac{\left(0.4 e^{i 0.9 \pi}, 0.4 e^{i 1.0 \pi}\right)}{(0,0)}, \frac{\left(0.7 e^{i 0.9 \pi}, 0.2 e^{i 0.5 \pi}\right)}{(0,1)}, \\
& \frac{\left(0.7 e^{i 0.3 \pi}, 0.2 e^{i 1.5 \pi}\right)}{(1,-1)}, \frac{\left(0.4 e^{i 0.3 \pi}, 0.4 e^{i 1.5 \pi}\right)}{(1,0)}, \\
& \left.\frac{\left(0.7 e^{i 0.3 \pi}, 0.2 e^{i 1.5 \pi}\right)}{(1,1)}\right\} .
\end{aligned}
$$


Definition 39. Let $A$ and $B$ be two complex Atanassov's intuitionistic fuzzy relations on $X \times Y$, where

$$
\begin{array}{r}
A=\left\{\left\langle(x, y), \mu_{A}(x, y)=r_{A}(x, y) e^{i \omega_{r_{A}}(x, y)},\right.\right. \\
\left.\left.\gamma_{A}(x, y)=k_{A}(x, y) e^{i \omega_{k_{A}}(x, y)}\right\rangle:(x, y) \in X \times Y\right\}, \\
B=\left\{\left\langle(x, y), \mu_{B}(x, y)=r_{B}(x, y) e^{i \omega_{r_{B}}(x, y)},\right.\right. \\
\left.\left.\gamma_{B}(x, y)=k_{B}(x, y) e^{i \omega_{k_{B}}(x, y)}\right\rangle:(x, y) \in X \times Y\right\} .
\end{array}
$$

The complex Atanassov's intuitionistic fuzzy union relation of $A$ and $B$, denoted by $A \cup B$, is specified by

$$
A \cup B\left\{\left\langle(x, y), \mu_{A \cup B}(x, y), \gamma_{A \cup B}(x, y)\right\rangle:(x, y) \in X \times Y\right\},
$$

where $\mu_{A \cup B}(x, y)=r_{A \cup B}(x, y) e^{i \omega_{r_{A \cup B}}(x, y)}=\max \left(r_{A}(x, y)\right.$, $\left.r_{B}(x, y)\right) e^{i \max \left(\omega_{r_{A}}(x, y), \omega_{r_{B}}(x, y)\right)}$ and $\gamma_{A \cup B}(x, y)=k_{A \cup B}(x$, $y) e^{i \omega_{k_{A \cup B}}(x, y)}=\min \left(k_{A}(x, y), k_{B}(x, y)\right) e^{i \min \left(\omega_{k_{A}}(x, y), \omega_{k_{B}}(x, y)\right)}$.

Example 40. Let $X=\{a, b\}$ and $Y=\{1,2\}$. Let

$$
\begin{aligned}
A= & \left\{\frac{\left(0.5 e^{i 1.3 \pi}, 0.4 e^{i 0.5 \pi}\right)}{(a, 1)}, \frac{\left(0.7 e^{i 0.3 \pi}, 0.2 e^{i 1.5 \pi}\right)}{(a, 2)},\right. \\
& \left.\frac{\left(1.0 e^{i 1.5 \pi}, 0.0 e^{i 0.5 \pi}\right)}{(b, 1)}, \frac{\left(0.8 e^{i 0.7 \pi}, 0.1 e^{i 1.1 \pi}\right)}{(b, 2)}\right\}, \\
B= & \left\{\frac{\left(0.8 e^{i 1.1 \pi}, 0.2 e^{i 0.7 \pi}\right)}{(a, 1)}, \frac{\left(0.7 e^{i 0.9 \pi}, 0.2 e^{i 0.4 \pi}\right)}{(a, 2)},\right. \\
& \left.\frac{\left(0.4 e^{i 0.9 \pi}, 0.4 e^{i \pi}\right)}{(b, 1)}, \frac{\left(0.1 e^{i \pi}, 0.7 e^{i 0.6 \pi}\right)}{(b, 2)}\right\} .
\end{aligned}
$$

Then,

$$
\begin{aligned}
A \cup B= & \left\{\frac{\left(0.8 e^{i 1.3 \pi}, 0.2 e^{i 0.5 \pi}\right)}{(a, 1)}, \frac{\left(0.7 e^{i 0.9 \pi}, 0.2 e^{i 0.4 \pi}\right)}{(a, 2)},\right. \\
& \left.\frac{\left(1.0 e^{i 1.5 \pi}, 0.0 e^{i 0.5 \pi}\right)}{(b, 1)}, \frac{\left(0.8 e^{i 1.0 \pi}, 0.1 e^{i 0.6 \pi}\right)}{(b, 2)}\right\} .
\end{aligned}
$$

Definition 41. Let $A$ and $B$ be two complex Atanassov's intuitionistic fuzzy relations on $X \times Y$, where

$$
\begin{gathered}
A=\left\{\left\langle(x, y), \mu_{A}(x, y)=r_{A}(x, y) e^{i \omega_{r_{A}}(x, y)},\right.\right. \\
\left.\left.\gamma_{A}(x, y)=k_{A}(x, y) e^{i \omega_{k_{A}}(x, y)}\right\rangle:(x, y) \in X \times Y\right\}, \\
B=\left\{\left\langle(x, y), \mu_{B}(x, y)=r_{B}(x, y) e^{i \omega_{r_{B}}(x, y)},\right.\right. \\
\left.\left.\gamma_{B}(x, y)=k_{B}(x, y) e^{i \omega_{k_{B}}(x, y)}\right\rangle:(x, y) \in X \times Y\right\} .
\end{gathered}
$$

The complex Atanassov's intuitionistic fuzzy intersection relation of $A$ and $B$, denoted by $A \cap B$, is specified by

$$
A \cap B\left\{\left\langle(x, y), \mu_{A \cap B}(x, y), \gamma_{A \cap B}(x, y)\right\rangle:(x, y) \in X \times Y\right\},
$$

where $\mu_{A \cap B}(x, y)=r_{A \cap B}(x, y) e^{i \omega_{r_{A \cap B}}(x, y)}=\min \left(r_{A}(x, y)\right.$, $\left.r_{B}(x, y)\right) e^{i \min \left(\omega_{r_{A}}(x, y), \omega_{r_{B}}(x, y)\right)}$ and $\gamma_{A \cap B}(x, y)=k_{A \cap B}(x$, $y) e^{i \omega_{k_{A \cap B}}(x, y)}=\max \left(k_{A}(x, y), k_{B}(x, y)\right) e^{i \max \left(\omega_{k_{A}}(x, y), \omega_{k_{B}}(x, y)\right)}$.

Example 42. Using the CAIFS $A$ and $B$ as in Example 40, we have

$$
\begin{aligned}
A \cap B= & \left\{\frac{\left(0.8 e^{i 1.3 \pi}, 0.2 e^{i 0.5 \pi}\right)}{(a, 1)}, \frac{\left(0.7 e^{i 0.9 \pi}, 0.2 e^{i 0.4 \pi}\right)}{(a, 2)},\right. \\
& \left.\frac{\left(1.0 e^{i 1.5 \pi}, 0.0 e^{i 0.5 \pi}\right)}{(b, 1)}, \frac{\left(0.8 e^{i 1.0 \pi}, 0.1 e^{i 0.6 \pi}\right)}{(b, 2)}\right\} .
\end{aligned}
$$

Following Zhang et al. [15], we call the relation $I$ an identical equal relation on $X$, if its membership and nonmembership functions are defined as follows:

$$
\begin{aligned}
& \mu_{I}(x, y)= \begin{cases}1 e^{i 2 \pi}=1 & \text { if } x=y \\
0 e^{i 0 \pi}=0 & \text { if } x \neq y,\end{cases} \\
& \gamma_{I}(x, y)= \begin{cases}0 e^{i 0 \pi}=0 & \text { if } x=y \\
1 e^{i 2 \pi}=1 & \text { if } x \neq y .\end{cases}
\end{aligned}
$$

Definition 43. Let $A \in \operatorname{CAIFS}(X \times X)$. If $\mu_{A}(x, x)=1$ and $\gamma_{A}(x, x)=0$ for all $x \in U$, then $A$ is a reflexive complex Atanassov's intuitionistic fuzzy relation.

Definition 44. Let $A \in \operatorname{CIFS}(X \times Y)$. Then $A^{T} \in \operatorname{CIFS}(Y \times X)$ is a replacement of $A$, where

$$
\begin{array}{r}
\mu_{A^{T}}(y, x)=\mu_{A}(x, y), \\
\gamma_{A^{T}}(y, x)=\gamma_{A}(x, y), \\
\forall(x, y) \in X \times Y .
\end{array}
$$

Proposition 45. If $A$ and $B$ are two complex Atanassov's intuitionistic fuzzy relations, then the following hold:
(1) $\left(A^{T}\right)^{T}=A$,
(2) $(A \cup B)^{T}=A^{T} \cup B^{T}$ and $(A \cap B)^{T}=A^{T} \cap B^{T}$,
(3) $(A \circ B)^{T}=B^{T} \circ A^{T}$ and $\left(A^{n}\right)^{T}=\left(A^{T}\right)^{n}$,
(4) $A \subseteq B$ if and only if $B^{T} \subseteq A^{T}$.

Proof. Trivial.

Definition 46. Let $A \in \operatorname{CIFS}(X \times Y)$. Then $A$ is a symmetrical complex Atanassov's intuitionistic fuzzy relation if

$$
\begin{array}{r}
\mu_{A}(x, y)=\mu_{A^{T}}(x, y), \\
\gamma_{A}(x, y)=\gamma_{A^{T}}(x, y), \\
\forall(x, y) \in X \times Y .
\end{array}
$$


Definition 47. Let $A \in \operatorname{CIFS}(X \times X)$. Then $A$ is a transitive complex Atanassov's intuitionistic fuzzy relation if $A \circ A \subseteq A$.

\section{Projection and Cylindric Extension of Complex Atanassov's Intuitionistic Fuzzy Relations}

In this section, we introduce the concepts of projection and cylindric extension as generalizations of projection and cylindric extension, respectively, for AIFRs.

Definition 48. Let $Q$ be a complex Atanassov's intuitionistic fuzzy relation on $X_{1} \times \cdots \times X_{n}$, and let $\left\{i_{1}, \ldots, i_{k}\right\}$ be a subsequence of $\{1, \ldots, n\}$. Then the projection of $Q$ on $X_{i_{1}} \times \cdots \times X_{i_{k}}$ is a complex Atanassov's intuitionistic fuzzy relation $Q_{p}$ on $X_{i_{1}} \times \cdots \times X_{i_{k}}$, defined by membership and nonmembership functions:

$$
\begin{aligned}
& \mu_{Q_{p}}\left(x_{i_{1}}, \ldots, x_{i_{k}}\right) \\
& =\max _{x_{j_{i}} \in X_{j_{1}}, \ldots, x_{j_{(n-k)}} \in X_{j_{(n-k)}}} r_{\mathrm{Q}}\left(x_{1}, \ldots, x_{n}\right) \\
& \cdot e^{i \max _{x_{j_{i}} \in X_{j_{1}}, \ldots, x_{j_{(n-k)}} \in X_{j_{(n-k)}} \omega_{r_{Q}}\left(x_{1}, \ldots, x_{n}\right)}}, \\
& \gamma_{Q_{p}}\left(x_{i_{1}}, \ldots, x_{i_{k}}\right) \\
& =\min _{x_{j_{i}} \in X_{j_{1}}, \ldots, x_{j_{(n-k)}} \in X_{j_{(n-k)}}} k_{Q}\left(x_{1}, \ldots, x_{n}\right) \\
& \cdot e^{i \min _{x_{j_{i}} \in X_{j_{1}}, \ldots, x_{j(n-k)} \in X_{j_{(n-k)}} \omega_{k}}\left(x_{1}, \ldots, x_{n}\right)},
\end{aligned}
$$

where the $\left\{x_{j_{1}}, \ldots, x_{j_{(n-k)}}\right\}$ is the complement of $\left\{x_{i_{1}}, \ldots, x_{i_{k}}\right\}$ with respect to $\left\{x_{1}, \ldots, x_{n}\right\}$.

Example 49. Let $X=\{2,3,4\}$ and $Y=\{a, b\}$ be two universes of discourse, and let $Q$ be a complex Atanassov's intuitionistic fuzzy relation on $X \times Y$ defined by

$$
\begin{aligned}
Q=\{ & \left\langle(2, a), 0.3 \cdot e^{i 0.9 \pi}, 0.5 \cdot e^{i 0.3 \pi}\right\rangle, \\
& \left\langle(3, a), 0.1 \cdot e^{i 1.5 \pi}, 0.7 \cdot e^{i \pi}\right\rangle, \\
& \left\langle(4, a), 0.5 \cdot e^{i 1.7 \pi}, 0.4 \cdot e^{i 0.5 \pi}\right\rangle, \\
& \left\langle(2, b), 0.5 \cdot e^{i 0.7 \pi}, 0.5 \cdot e^{i 2 \pi}\right\rangle, \\
& \left\langle(3, b), 0.3 \cdot e^{i 0.2 \pi}, 0.4 \cdot e^{i 1.7 \pi}\right\rangle, \\
& \left.\left\langle(4, b), 0.3 \cdot e^{i 1.5 \pi}, 0.4 \cdot e^{i 1.3 \pi}\right\rangle\right\} .
\end{aligned}
$$

We compute the projection of CAIFR $Q$ on $X$ and $Y$, denoted by $Q_{X}$ and $Q_{Y}$ respectively. The projection $Q_{X}$, is given by

$$
\begin{aligned}
& \mu_{Q_{p}}(x)=\left(\max _{y \in Y} r_{Q}(x, y)\right) \cdot e^{i \max _{y \in Y} \omega_{r_{Q}}(x, y)}, \\
& \gamma_{Q_{p}}(x)=\left(\min _{y \in Y} k_{Q}(x, y)\right) \cdot e^{i \min _{y \in Y} \omega_{k_{Q}}(x, y)} .
\end{aligned}
$$

$$
\begin{aligned}
Q_{X}(x)=\{ & \left\langle 2, \vee(0.3,0.5) e^{i \vee(0.9 \pi, 0.7 \pi)},\right. \\
& \left.\wedge(0.5,0.5) e^{i \wedge(0.3 \pi, 2 \pi)}\right\rangle, \\
& \left\langle 3, \vee(0.1,0.3) e^{i \vee(1.5 \pi, 0.2 \pi)},\right. \\
& \left.\wedge(0.7,0.4) e^{i \wedge(\pi, 1.7 \pi)}\right\rangle, \\
& \left\langle 4, \vee(0.5,0.3) e^{i \vee(1.7 \pi, 1.5 \pi)},\right. \\
& \left.\left.\wedge(0.4,0.4) e^{i \wedge(0.5 \pi, 1.3 \pi)}\right\rangle\right\} . \\
= & \left\langle 2,0.5 e^{i 0.9 \pi}, 0.5 e^{i 0.3 \pi}\right\rangle,\left\langle 3,0.3 e^{i 1.5 \pi}, 0.4 e^{i \pi}\right\rangle, \\
& \left.\left\langle 4,0.5 e^{i 1.7 \pi}, 0.4 e^{i 0.5 \pi}\right\rangle\right\} .
\end{aligned}
$$

The projection $Q_{Y}$ is given by

$$
\begin{aligned}
& \mu_{\mathrm{Q}_{Y}}(y)=\left(\max _{x \in X} r_{\mathrm{Q}}(x, y)\right) \cdot e^{i \max _{x \in X} \omega_{r}(x, y)}, \\
& \gamma_{\mathrm{Q}_{Y}}(x)=\left(\min _{x \in X} k_{\mathrm{Q}}(x, y)\right) \cdot e^{i \min _{\mathrm{x} \in \mathrm{X}} \omega_{k}(x, y)} \cdot \\
& Q_{Y}(y)=\left\{\left\langlea, \vee(0.3,0.1,0.5) e^{i \vee(0.9 \pi, 1.5 \pi, 1.7 \pi)},\right.\right. \\
&\left.\wedge(0.5,0.7,0.4) e^{i \wedge(0.3 \pi, \pi, 0.5 \pi)}\right\rangle, \\
&\left\langle b, \vee(0.5,0.3,0.3) e^{i \vee(0.7 \pi, 0.2 \pi, 1.5 \pi)},\right. \\
&\left.\left.\wedge(0.5,0.4,0.4) e^{i \wedge(2 \pi, 1.7 \pi, 1.3 \pi)}\right\rangle\right\} . \\
&=\left\{\left\langle a, 0.5 e^{i 1.7 \pi}, 0.4 e^{i 0.3 \pi}\right\rangle,\left\langle b, 0.5 \cdot e^{i 1.5 \pi}, 0.4 e^{i 1.3 \pi}\right\rangle\right\} .
\end{aligned}
$$

Definition 50. Let $Q$ be a complex Atanassov's intuitionistic fuzzy relation on $X_{1} \times \cdots \times X_{n}$ and let $\left\{i_{1}, \ldots, i_{k}\right\}$ be a subsequence of $\{1, \ldots, n\}$. The cylindric extension of $Q_{P}$ to $X_{1} \times \cdots \times X_{n}$, is a complex Atanassov's intuitionistic fuzzy relation $Q_{\mathrm{PE}}$ on $X_{1} \times \cdots \times X_{n}$, defined by membership and nonmembership functions:

$$
\begin{aligned}
& \mu_{\mathrm{QPE}_{\mathrm{PE}}}\left(x_{1}, \ldots, x_{n}\right)=\left[r_{\mathrm{Q}_{P}}\left(x_{i_{1}}, \ldots, x_{i_{k}}\right) \cdot e^{i \omega_{\mathrm{r}_{P}}\left(x_{i_{1}}, \ldots, x_{i_{k}}\right)}\right], \\
& \gamma_{\mathrm{Q}_{\mathrm{PE}}}\left(x_{1}, \ldots, x_{n}\right)=\left[k_{\mathrm{Q}_{P}}\left(x_{i_{1}}, \ldots, x_{i_{k}}\right) \cdot e^{i \omega_{k_{Q_{P}}}\left(x_{i_{1}}, \ldots, x_{i_{k}}\right)}\right] .
\end{aligned}
$$

Example 51. We consider Example 49 to find the cylindric extensions of $Q_{X}$ and $Q_{Y}$. The cylindric extension of $Q_{X}$ is a complex Atanassov's intuitionistic fuzzy relation $Q_{X E}$ given by $\mu_{\mathrm{Q}_{\mathrm{XE}}}(x, y)=\mu_{\mathrm{Q}_{\mathrm{X}}}(x)$, and $\gamma_{\mathrm{Q}_{\mathrm{XE}}}(x, y)=\gamma_{\mathrm{Q}_{X}}(x)$. Then,

$$
\begin{aligned}
Q_{\mathrm{XE}}=\{ & \left\langle(2, a), 0.5 e^{i 0.9 \pi}, 0.5 e^{i 0.3 \pi}\right\rangle, \\
& \left\langle(3, a), 0.3 e^{i 1.5 \pi}, 0.4 e^{i \pi}\right\rangle, \\
& \left\langle(4, a), 0.5 e^{i 1.7 \pi}, 0.4 e^{i 0.5 \pi}\right\rangle, \\
& \left\langle(2, b), 0.5 e^{i 0.9 \pi}, 0.5 e^{i 0.3 \pi}\right\rangle, \\
& \left\langle(3, b), 0.3 e^{i 1.5 \pi}, 0.4 e^{i \pi}\right\rangle, \\
& \left.\left\langle(4, b), 0.5 e^{i 1.7 \pi}, 0.4 e^{i 0.5 \pi}\right\rangle\right\} .
\end{aligned}
$$


The cylindric extension of $Q_{Y}$ is a complex Atanassov's intuitionistic fuzzy relation $Q_{\mathrm{YE}}$ given by $\mu_{\mathrm{Q}_{\mathrm{YE}}}(x, y)=$ $\mu_{\mathrm{Q}_{Y}}(y)$, and $\gamma_{\mathrm{Q}_{\mathrm{YE}}}(x, y)=\gamma_{\mathrm{Q}_{Y}}(y)$. Thus,

$$
\begin{aligned}
Q_{\mathrm{YE}}=\{ & \left\langle(2, a), 0.5 e^{i 1.7 \pi}, 0.4 e^{i 0.3 \pi}\right\rangle, \\
& \left\langle(3, a), 0.5 e^{i 1.7 \pi}, 0.4 e^{i 0.3 \pi}\right\rangle, \\
& \left\langle(4, a), 0.5 e^{i 1.7 \pi}, 0.4 e^{i 0.3 \pi}\right\rangle, \\
& \left\langle(2, b), 0.5 e^{i 1.5 \pi}, 0.4 e^{i 1.3 \pi}\right\rangle, \\
& \left\langle(3, b), 0.5 e^{i 1.5 \pi}, 0.4 e^{i 1.3 \pi}\right\rangle, \\
& \left.\left\langle(4, b), 0.5 e^{i 1.5 \pi}, 0.4 e^{i 1.3 \pi}\right\rangle\right\} .
\end{aligned}
$$

\section{Composition of Complex Atanassov's Intuitionistic Fuzzy Relations}

In this section, we introduce the definition of composition of complex Atanassov's intuitionistic fuzzy relations. Some properties of composition of CAIFRs are obtained. The composition of complex Atanassov's intuitionistic fuzzy relations is introduced as in the following.

Definition 52. Let $X, Y$, and $Z$ be universes, $A$ a complex Atanassov's intuitionistic fuzzy relation of $X$ and $Y$, and $B$ a complex Atanassov's intuitionistic fuzzy relation of $Y$ and $Z$. The composition of $A$ and $B$, denoted by $A \circ B$, is a complex Atanassov's intuitionistic fuzzy relation of $X$ and $Z$, specified by the following functions:

$$
\begin{aligned}
\mu_{A \circ B}(x, z)= & r_{A \circ B}(x, z) \cdot e^{i \omega_{r A \circ B}(x, z)} \\
= & {\left[\sup _{y \in Y} \min \left(r_{A}(x, y), r_{B}(y, z)\right)\right.} \\
& \left.\cdot e^{i \sup _{y \in Y} \min \left(\omega_{r A}(x, y), \omega_{r B}(y, z)\right)}\right], \\
\gamma_{A \circ B}(x, z)= & \left.r_{A \circ B}(x, y) \cdot e^{i \omega_{k A \circ B}(x, z)}\right] \\
= & {\left[\inf _{y \in Y} \max \left(k_{A}(x, y), k_{B}(y, z)\right)\right.} \\
& \left.\cdot e^{i \inf _{y \in Y} \max \left(\omega_{k A}(x, y), \omega_{k B}(y, z)\right)}\right] .
\end{aligned}
$$

Example 53. Let $K, S, J, P$, and $L$ be name of cities, and let $X=\{K, S\}, Y=\{K, J\}$ and $W=\{P, L\}$ be three sets of these cities. Then, the Cartesian product of $X$ and $Y$ is the set $X \times$ $Y=\{(K, K),(K, J),(S, K),(S, J)\}$, and the Cartesian product of $Y$ and $Z$ is the set $Y \times Z=\{(K, P),(K, L),(J, P),(J, L)\}$. For example, let $A(X, Y)$ be a relation called "the first element (city) is colder than the second element (city) in winter, summer, autumn, or spring," and let $B(Y, Z)$ be a relation called "the first element (city) is more attractive than the second element (city) in winter, summer, autumn or spring."
The relations $A(X, Y)$ and $B(Y, Z)$ can be presented by the following relational matrices:

$$
\begin{aligned}
& A(X, Y) \\
& \quad=\left[\begin{array}{ccc}
K & \left(0.9 e^{i(0.3 / 24) \pi}, 0 e^{i(3 / 24) \pi}\right) & \left(0.7 e^{i(1.5 / 24) \pi}, 0.3 e^{i(5 / 24) \pi}\right) \\
S & \left(0.6 e^{i(1 / 24) \pi}, 0.3 e^{i(1.8 / 24) \pi}\right) & \left(0.3 e^{i(3 / 24) \pi}, 0.7 e^{i(1 / 24) \pi}\right)
\end{array}\right], \\
& B(Y, Z) \\
& \quad=\left[\begin{array}{ccc}
K & \left(0.2 e^{i(3 / 24) \pi}, 0.7 e^{i(3 / 24) \pi}\right) & \left(0.1 e^{i(12 / 24) \pi}, 0.8 e^{i(5 / 24) \pi}\right) \\
J & \left(0.1 e^{i(15 / 24) \pi}, 0.9 e^{i(5 / 24) \pi}\right) & \left(0.2 e^{i(3 / 24) \pi}, 0.6 e^{i(2 / 24) \pi}\right)
\end{array}\right] .
\end{aligned}
$$

We compute the composite relational matrix, denoted by $A \circ B(X, Z)$. We should note that $X \times Z$ has four elements: $(K, P),(K, L),(S, P)$, and $(S, L)$. Thus, our task is to determine the membership, $\mu_{A \circ B}$, and nonmembership, $\gamma_{A \circ B}$, values for the four elements above. We use Definition 52 to determine the membership and nonmembership of $A$ 。 $B(K, P)$. Thus, we have

$$
\begin{aligned}
\mu_{A \circ B}(K, P) & \\
= & \sup _{y \in V}\left\{\min \left[\left(r_{A}(K, K)\right), r_{B}(K, P)\right],\right. \\
& \left.\min \left[\left(r_{A}(K, J)\right), r_{B}(J, P)\right]\right\} \\
& \cdot e^{\sup _{y \in V}\left\{\min \left[\left(\omega_{r_{A}}(K, K)\right), \omega_{r_{B}}(K, P)\right], \min \left[\left(\omega_{r_{A}}(K, J)\right), \omega_{r_{B}}(J, P)\right]\right\}} \\
= & \sup _{y \in V}\{\min [0.9,0.2], \min [0.7,0.1]\} \\
& \cdot e^{i \sup _{y \in V}\{\min [(0.3 / 24) \pi,(3 / 24) \pi], \min [(1.5 / 24) \pi,(15 / 24) \pi]\}} \\
= & \sup _{y \in V}\{0.2,0.1\} e^{i \sup _{y \in V}\{(0.3 / 24) \pi,(1.5 / 24) \pi\}}=0.2 e^{i(1.5 / 24) \pi} .
\end{aligned}
$$

Analogously, we have

$$
\begin{aligned}
& \gamma_{A \circ B}(K, P) \\
& =\inf _{y \in V}\left\{\max \left[\left(k_{A}(K, K)\right), k_{B}(K, P)\right],\right. \\
& \left.\max \left[\left(k_{A}(K, J)\right), k_{B}(J, P)\right]\right\} \\
& \cdot e^{\inf _{y \in V}\left\{\max \left[\left(\omega_{k_{A}}(K, K)\right), \omega_{k_{B}}(K, P)\right], \max \left[\left(\omega_{k_{A}}(K, J)\right), \omega_{k_{B}}(J, P)\right]\right\}} \\
& =\inf _{y \in V}\{\max [0,0.7], \max [0.3,0.9]\}
\end{aligned}
$$

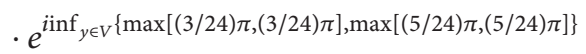

$$
\begin{aligned}
& =\inf _{y \in V}\{0.7,0.9\} e^{\left.i \inf _{y \in V}\{(3 / 24) \pi,(5 / 24) \pi]\right\}}=0.7 e^{i(3 / 24) \pi} .
\end{aligned}
$$


Analogously, we determine the elements $(K, L),(S, P)$, and $(S, L)$. Thus, the final relational matrix of $A \circ B$ is

$$
\begin{aligned}
& A \circ B(X, Z) \\
& =\left[\begin{array}{ccc}
P & L \\
K & \left(0.2 e^{i(15 / 24) \pi}, 0.7 e^{i(3 / 24) \pi}\right) & \left(0.2 e^{i(3 / 24) \pi}, 0.6 e^{i(5 / 24) \pi}\right) \\
S & \left(0.2 e^{i(3 / 24) \pi}, 0.7 e^{i(5 / 24) \pi}\right) & \left(0.2 e^{i(3 / 24) \pi}, 0.7 e^{i(2 / 24) \pi}\right)
\end{array}\right] .
\end{aligned}
$$

Note 2. In Example 53 the amplitude terms represent the belongingness and nonbelongingness to the set of cold and attractiveness, and the phase terms represent the belongingness and nonbelongingness to the phase of season.

\section{Max-Min and Max Product Compositions}

(1) The max-min composition of CAIFRs between $A(X, Y)$ and $B(Y, Z)$ is a CAIFR $A \circ B$ in $X \times Z$, defined by the membership and nonmembership functions

$$
\begin{aligned}
\mu_{A \circ B}(x, z)= & {\left[\max _{y \in V} \min \left(r_{A}(x, y), r_{A}(y, z)\right)\right.} \\
& \left.\cdot e^{i \max _{y \in V} \min \left(\arg _{\mu A}(x, y), \arg _{\mu B}(y, z)\right)}\right], \\
\gamma_{A \circ B}(x, z)= & {\left[\min _{y \in V} \max \left(r_{A}(x, y), r_{B}(y, z)\right)\right.} \\
& \left.\cdot e^{i \min _{y \in V} \max \left(\arg _{\gamma A}(x, y), \arg _{\gamma B}(y, z)\right)}\right] .
\end{aligned}
$$

(2) The max product composition of CAIFRs between $A(X, Y)$ and $B(Y, Z)$ is a CAIFR $A \circ B$ in $X \times Z$, defined by the membership and nonmembership functions:

$$
\begin{gathered}
\mu_{A \circ B}(x, z)=\left[\max _{y \in V}\left(r_{A}(x, y) \cdot r_{B}(y, z)\right)\right. \\
\left.\cdot e^{i(1 / 2 \pi) \max _{y \in V}\left(\arg _{\mu A}(x, y) \cdot \arg _{\mu B}(y, z)\right)}\right], \\
\mu_{A \circ B}(x, z)=\left[\min _{y \in V}\left(r_{A}(x, y) \cdot r_{B}(y, z)\right)\right. \\
\left.\cdot e^{i(1 / 2 \pi) \min _{y \in V}\left(\arg _{\gamma A}(x, y) \cdot \arg _{\gamma B}(y, z)\right)}\right] .
\end{gathered}
$$

Proposition 54. Let $W, X, Y$, and $Z$ be universes, $A$ a complex Atanassov's intuitionistic fuzzy relation of $W$ and $X, B$ a complex Atanassov's intuitionistic fuzzy relation of $X$ and $Y$, and $C$ a complex Atanassov's intuitionistic fuzzy relation of $Y$ and $Z$. Then $A \circ(B \circ C)=(A \circ B) \circ C$.

Proof. Let $A \circ(B \circ C)=\left\{\left\langle(w, z), \mu_{A \circ(B \circ C)}(w, z), \gamma_{A \circ(B \circ C)}(w, z)\right\rangle\right.$ : $(w, z) \in W \times Z\}$, and $(A \circ B) \circ C=\{\langle(w, z)$, $\left.\left.\mu_{(A \circ B) \circ C}(w, z), \gamma_{(A \circ B) \circ C}(w, z)\right\rangle:(w, z) \in W \times Z\right\}$. To prove the equality $A \circ(B \circ C)=(A \circ B) \circ C$, we have to show that
$\mu_{A \circ(B \circ C)}=\mu_{(A \circ B) \circ C}$ and $\gamma_{A \circ(B \circ C)}=\gamma_{(A \circ B) \circ C}$. By Definition 52, we have

$$
\begin{aligned}
& \mu_{A \circ(B \circ C)}(w, z) \\
& =\left[\sup _{x \in X} \min \left[r_{A}(w, x),\left(r_{(B \circ C)}(x, z)\right)\right]\right. \\
& \left.\cdot e^{i \sup _{x \in X} \min \left[\omega_{\mu_{A}}(w, x),\left(\omega_{\mu_{(B \circ C)}}(x, z)\right)\right]}\right] \\
& =\left[\sup _{x \in X} \min \left[r_{A}(w, x),\left(\sup _{y \in Y} \min \left(r_{B}(x, y), r_{C}(y, z)\right)\right)\right]\right. \\
& \left.\cdot e^{i \sup _{x \in X} \min \left[\omega_{\mu_{A}}(w, x),\left(\sup _{y \in Y} \min \left(\omega_{\mu_{B}}(x, y), \omega_{\mu_{C}}(y, z)\right)\right)\right]}\right] \\
& =\left[\sup _{x \in X, y \in Y} \min \left[r_{A}(w, x), r_{B}(x, y), r_{C}(y, z)\right]\right. \\
& \left.\cdot e^{i \sup _{x \in X, y \in Y} \min \left[\omega_{\mu_{A}}(w, x), \omega_{\mu_{B}}(x, y), \omega_{\mu_{C}}(y, z)\right]}\right] \\
& =\left[\sup _{y \in Y} \min \left[\left(\sup _{x \in X} \min \left(r_{A}(w, x), r_{B}(x, y)\right)\right), r_{C}(y, z)\right]\right. \\
& \left.\cdot e^{i \sup _{y \in Y} \min \left[\left(\sup _{x \in X} \min \left(\omega_{\mu_{A}}(w, x), \omega_{\mu_{B}}(x, y)\right)\right), \omega_{\mu_{C}}(y, z)\right]}\right] \\
& =\left[\sup _{y \in Y} \min \left[\left(r_{(A \circ B)}(w, y)\right), r_{C}(y, z)\right]\right. \\
& \left.\cdot e^{i \sup _{y \in Y} \min \left[\left(\omega_{\mu_{(A \circ B)}}(w, y)\right), \omega_{\mu_{C}}(y, z)\right]}\right]=\mu_{(A \circ B) \circ C}(w, z) .
\end{aligned}
$$

Also,

$$
\begin{aligned}
& \gamma_{A \circ(B \circ C)}(w, z) \\
& =\left[\inf _{x \in X} \max \left[k_{A}(w, x),\left(k_{(B \circ C)}(x, z)\right)\right]\right. \\
& \left.\cdot e^{i \inf _{x \in X} \max \left[\omega_{\gamma_{A}}(w, x),\left(\omega_{\gamma_{(B \circ C)}}(x, z)\right)\right]}\right] \\
& =\left[\operatorname { i n f } _ { x \in X } \operatorname { m a x } \left[k_{A}(w, x),\right.\right. \\
& \left.\left(\inf _{y \in Y} \max \left(k_{B}(x, y), k_{C}(y, z)\right)\right)\right] \\
& \left.\cdot e^{i \inf f_{x \in X} \max \left[\omega_{\gamma_{A}}(w, x),\left(\inf _{y \in Y} \max \left(\omega_{\gamma_{B}}(x, y), \omega_{\gamma_{C}}(y, z)\right)\right)\right]}\right] \\
& =\left[\inf _{x \in X, y \in Y}\left[k_{A}(w, x), k_{B}(w, y), k_{C}(y, z)\right]\right. \\
& \left.\cdot e^{i \inf f_{x \in X, y \in Y} \max \left[\omega_{\gamma_{A}}(w, x), \omega_{\gamma_{B}}(w, y), \omega_{\gamma_{C}}(y, z)\right]}\right]
\end{aligned}
$$




$$
\begin{aligned}
= & \inf _{y \in Y} \max \left[\left(\inf _{x \in X} \max \left(k_{A}(w, x), k_{B}(x, y)\right)\right),\right. \\
& \left.k_{C}(y, z)\right] \\
& \left.\cdot e^{i \inf _{y \in Y} \max }\right] \\
= & {\left[\inf _{y \in Y} \max \left[\left(k_{(A \circ B)}(w, y)\right), k_{C}(y, z)\right]\right.} \\
& \left.\cdot e^{i \inf _{y \in Y} \max \left[\left(\omega_{\gamma_{(A \circ B)}}(w, y)\right), \omega_{\gamma_{C}}(y, z)\right]}\right]=\gamma_{(A \circ B) \circ C}(w, z) .
\end{aligned}
$$

So we have $\mu_{A \circ(B \circ C)}=\mu_{(A \circ B) \circ C}$ from (82), and we have $\gamma_{A \circ(B \circ C)}=\gamma_{(A \circ B) \circ C}$ from (83). Therefore, the proof is completed.

Proposition 55. Let $X, Y$, and $Z$ be universes, $A$ and $B$ two complex Atanassov's intuitionistic fuzzy relations of $X$ and $Y$, and $C$ a complex Atanassov's intuitionistic fuzzy relation of $Z$ and $X$. Then,

(i) $C \circ(A \cup B)=(C \circ A) \cup(C \circ B)$,

(ii) $C \circ(A \cap B) \subseteq(C \circ A) \cap(C \circ B)$.

Proof. (i) To prove the equality $C \circ(A \cup B)=(C \circ A) \cup(C \circ B)$, we have to show that $\mu_{C \circ(A \cup B)}=\mu_{(C \circ A) \cup(C \circ B)}$ and $\gamma_{C \circ(A \cup B)}=$ $\gamma_{(C \circ A) \cup(C \circ B)}$. By Definitions 52, 17, and 18 we have

$$
\begin{aligned}
\mu_{C \circ}(A \cup B)= & \sup \min \left[r_{C},\left(r_{(A \cup B)}\right)\right] \cdot e^{i \sup \min \left[\omega_{\mu_{C}},\left(\omega_{\mu_{(A \cup B)}}\right)\right]} \\
= & \sup \min \left[r_{C},\left(\max \left(r_{A}, r_{B}\right)\right)\right] \\
& \cdot e^{i \sup \min \left[\omega_{\mu_{C}},\left(\max \left(\omega_{\mu_{A}}, \omega_{\mu_{B}}\right)\right)\right]} \\
= & \max \left[\left(\sup \min \left(r_{C}, r_{A}\right)\right),\left(\sup \min \left(r_{C}, r_{B}\right)\right)\right] \\
& \cdot e^{i \max \left[\left(\sup \min \left(\omega_{\mu_{C}}, \omega_{\mu_{A}}\right)\right),\left(\sup \min \left(\omega_{\mu_{C}}, \omega_{\mu_{B}}\right)\right)\right]} \\
= & \mu_{(C \circ A) \cup(C \circ B)},
\end{aligned}
$$

Also,

$$
\begin{aligned}
& \gamma_{C \circ(A \cup B)} \\
&=\inf \max \left[k_{C},\left(k_{(A \cup B)}\right)\right] \cdot e^{\inf \max \left[\omega_{\gamma_{C}},\left(\omega_{\gamma_{(A \cup B)}}\right)\right]} \\
&=\left[\inf \max \left[k_{C},\left(\min \left(k_{A}, k_{B}\right)\right)\right]\right. \\
&\left.\cdot e^{\inf \max \left[\omega_{\gamma_{C}},\left(\min \left(\omega_{\gamma_{A}}, \omega_{\gamma_{B}}\right)\right)\right]}\right] \\
&=\left[\min \left[\left(\inf \max \left(k_{C}, k_{A}\right)\right),\left(\inf \max \left(k_{C}, k_{B}\right)\right)\right]\right. \\
&\left.\cdot e^{\min \left[\left(\inf \max \left(\omega_{\gamma_{C}}, \omega_{\gamma_{A}}\right)\right), \inf \left(\max \left(\omega_{\gamma_{C}}, \omega_{\gamma_{B}}\right)\right)\right]}\right] \\
&= \gamma_{(C \circ A) \cup(C \circ B)} .
\end{aligned}
$$

Therefore, by (84) and (85), proof of (i) is completed. (ii) To prove the subsets $C \circ(A \cap B) \subseteq(C \circ A) \cap$ $(C \circ B)$, we have to show that $r_{C \circ(A \cap B)} \leq r_{(C \circ A) \cap(C \circ B)}$ and $\operatorname{Arg}_{\mu_{C \circ(A \cap B)}} \geq \operatorname{Arg}_{\mu_{(\mathrm{C} \circ A) \cap(C \circ B)}}$ for the membership function and $k_{C \circ(A \cap B)} \leq k_{(C \circ A) \cap(C \circ B)}$ and $\operatorname{Arg}_{\gamma_{C \circ(A \cap B)}} \leq \operatorname{Arg}_{\gamma_{(C \circ A) \cap(C \circ B)}}$ for the nonmembership function. By Definitions 28, 52, and 18, we have

$$
\begin{aligned}
r_{C \circ(A \cap B)} & =\sup \min \left[r_{C}, \min \left(r_{A}, r_{B}\right)\right]=\sup \left(r_{j}\right) \\
r_{(C \circ A) \cap(C \circ B)} & =\min \left[\operatorname{supmin}\left(r_{C}, r_{A}\right), \sup \min \left(r_{C}, r_{B}\right)\right] \\
& =\min \sup \left(r_{j}\right),
\end{aligned}
$$

where $j=A, B$, or $C$ for any of the following cases:

$$
\begin{array}{lll}
r_{A} \leq r_{B} \leq r_{C}, & r_{B} \leq r_{A} \leq r_{C}, & r_{C} \leq r_{B} \leq r_{A}, \\
r_{C} \leq r_{A} \leq r_{B}, & r_{A} \leq r_{C} \leq r_{B}, & r_{B} \leq r_{C} \leq r_{A} .
\end{array}
$$

So, we conclude that

$$
r_{C \circ(A \cap B)}=\sup \left(r_{j}\right) \geq \min \sup \left(r_{j}\right)=r_{(C \circ A) \cap(C \circ B)} .
$$

To prove the nonmembership parts, we use similar steps as for the membership term. Therefore, this completes the proof of (ii).

Corollary 56. Let $X, Y$, and $Z$ be universes, $A_{t}(t \in T)$ complex Atanassov's intuitionistic fuzzy relations of $X$ and $Y$, and $C$ a complex Atanassov's intuitionistic fuzzy relation of $Z$ and $X$, where $T$ is an arbitrary index set. Then,

(i) $C \circ\left(\cup_{t \in T} A_{t}\right)=\cup_{t \in T}\left(C \circ A_{t}\right)$,

(ii) $C \circ\left(\cap_{t \in T} A_{t}\right) \subseteq \cap_{t \in T}\left(C \circ A_{t}\right)$.

Proof. This is straightforward from Proposition 55.

Proposition 57. Let $X, Y$, and $Z$ be universes, $A$ and $B$ two complex Atanassov's intuitionistic fuzzy relations of $X$ and $Y$, and $C$ a complex fuzzy relation of $Z$ and $X$. If $A \subseteq B$, then

(i) $C \circ A \subseteq C \circ B$.

Proof. This is straightforward from Proposition 55 and Definitions 52 and 28.

\section{Conclusions}

In this research, a new model deals with multi-attribute decision-making problems by representing the type of Atanassov's intuitionistic fuzzy information which is happening repeatedly over a period of time. This generalised model (Application 31) is easy to realize and appropriate with human recognition. The difference between this model and Atanassov's intuitionistic fuzzy model in [12] is that in our approach, complex Atanassov's intuitionistic fuzzy set represents the problems with Atanassov's intuitionistic fuzzy uncertainty and periodicity simultaneously, in which the phase degree, of membership and nonmembership have 
precious and pivotal values. Besides, the phase term can represent both the fuzzy information and precise/accurate time. So, CAIFSs can be useful and applicable in many areas, such as decision-making processes, to select the best alternative.

The development of complex Atanassov's intuitionistic fuzzy relation is important as it forms a basis for the derivation of complex Atanassov's intuitionistic fuzzy logic. Complex Atanassov's intuitionistic fuzzy relation is formally generalised from a conventional Atanassov's intuitionistic fuzzy relation, based on complex Atanassov's intuitionistic fuzzy sets, in which the ranges of values of CAIFR are extended to the unit circle in complex plane instead of $[0,1]$ as in the conventional Atanassov's intuitionistic fuzzy functions. We have also introduced the Cartesian product of CAIFSs and derived two properties of the product space. The concepts of projection, cylindric extension, and composition of CAIFRs are also introduced. Finally, an example of CAIFR in real life situation is illustrated in this paper.

\section{Future Research}

In future research, it is a meaningful topic to introduce the concept of CAIF logics and systems based on CAIF relation, which gives the ability to extend the realm of reallife applications in the CAIF environment. The concepts of logics and systems open for us immense applications in engineering, medical, physics, automobiles, defense and security, internet and computer security, and other fields. We can also introduce $\delta$-equalities of complex Atanassov's intuitionistic fuzzy relations.

\section{Acknowledgment}

The work presented in this paper was supported by Universiti Kebangsaan Malaysia under Grant no. UKM-DLP-2011-038.

\section{References}

[1] K. T. Atanassov, "Intuitionistic fuzzy sets," Fuzzy Sets and Systems, vol. 20, no. 1, pp. 87-96, 1986.

[2] L. A. Zadeh, "Fuzzy sets," Information and Computation, vol. 8, pp. 338-353, 1965.

[3] M. Fathi, On Intuitionistic fuzzy sets, MSc Research Project, Faculty of Science and Technology, Universiti Kebangsaan Malaysia, 2007.

[4] D. Ramot, R. Milo, M. Friedman, and A. Kandel, "Complex fuzzy sets," IEEE Transactions on Fuzzy Systems, vol. 10, no. 2, pp. 171-186, 2002.

[5] M. Jun, G. Zhang, and J. Lu, "A Method for multiple periodic factor prediction problems using complex fuzzy sets," IEEE Transactions on Fuzzy Systems, vol. 20, no. 1, pp. 32-45, 2012.

[6] Z. Chen, S. Aghakhani, J. Man, and S. Dick, "ANCFIS: a neurofuzzy architecture employing complex fuzzy sets," IEEE Transactions on Fuzzy Systems, vol. 19, no. 2, pp. 305-322, 2011.

[7] G. Wei and X. Zhao, "Some induced correlated aggregating operators with intuitionistic fuzzy information and their application to multiple attribute group decision making," Expert Systems with Applications, vol. 39, no. 2, pp. 2026-2034, 2012.
[8] Z. Pei and L. Zheng, "A novel approach to multi-attribute decision making based on intuitionistic fuzzy sets," Expert Systems with Applications, vol. 39, no. 3, pp. 2560-2566, 2012.

[9] Z. X. Su, G. P. Xia, M. Y. Chen, and L. Wang, "Induced generalized intuitionistic fuzzy OWA operator for multi-attribute group decision making," Expert Systems with Applications, vol. 39, no. 2, pp. 1902-1910, 2012.

[10] C. L. Hawang and K. Yoon, Lecture Notes in Economics and Mathematics Systems, Springer, Heidelberg, Germany, 1981.

[11] A. Alkouri and A. Salleh, "Complex intuitionistic fuzzy sets," in International Conference on Fundamental and Applied Sciences, AIP Conference Proceedings, vol. 1482, pp. 464-470, 2012.

[12] Y. Song, Q. Zhang, and X. Zhou, "Supplier selection model based on distance measure between intuitionistic fuzzy sets," in Proceedings of the IEEE International Conference on Systems, Man and Cybernetics (SMC '06), pp. 3795-3799, Taipei, Taiwan, October 2006.

[13] J. J. Buckley, "Fuzzy complex numbers," Fuzzy Sets and Systems, vol. 33, no. 3, pp. 333-345, 1989.

[14] H. T. Nguyen, A. Kandel, and V. Kreinovich, "Complex fuzzy sets: towards new foundations," in Proceedings of the 9th IEEE International Conference on Fuzzy Systems, pp. 1045-1048, May 2000.

[15] G. Zhang, T. S. Dillon, K. Y. Cai, J. Ma, and J. Lu, " $\delta$-equalities of complex fuzzy relations," in Proceedings of the 24th IEEE International Conference on Advanced Information Networking and Applications (AIN '10), pp. 1218-1224, April 2010.

[16] G. Zhang, T. S. Dillon, K.-Y. Cai, J. Ma, and J. Lu, "Operation properties and $\delta$-equalities of complex fuzzy sets," International Journal of Approximate Reasoning, vol. 50, no. 8, pp. 1227-1249, 2009.

[17] A. Di Nola, W. Pedrycz, and S. Sessa, "Fuzzy relation equations with equality and difference composition operators," Fuzzy Sets and Systems, vol. 25, no. 2, pp. 205-215, 1988.

[18] A. Di Nola, W. Pedrycz, S. Sessa, and E. Sanchez, "Fuzzy relation equations theory as a basis of fuzzy modelling: an overview," Fuzzy Sets and Systems, vol. 40, no. 3, pp. 415-429, 1991.

[19] W. Bandler and L. J. Kohout, "Special properties, closures and interiors of crisp and fuzzy relations," Fuzzy Sets and Systems, vol. 26, no. 3, pp. 317-331, 1988.

[20] J. M. Mendel, "Fuzzy logic systems for engineering: a tutorial," Proceedings of the IEEE, vol. 83, no. 3, pp. 345-377, 1995.

[21] H. Bustince, "Construction of intuitionistic fuzzy relations with predetermined properties," Fuzzy Sets and Systems, vol. 109, no. 3, pp. 379-403, 2000.

[22] H. Bustince and P. Burillo, "Structures on intuitionistic fuzzy relations," Fuzzy Sets and Systems, vol. 78, no. 3, pp. 293-303, 1996.

[23] P. Burillo and H. Bustince, "Intuitionistic fuzzy relations I," Mathware \& Soft Computing, vol. 2, no. 1, pp. 5-38, 1995.

[24] P. Burillo and H. Bustince, "Bustince intuitionistic fuzzy relations II," Mathware \& Soft Computing, vol. 2, no. 2, pp. 117-148, 1995.

[25] F. Adam, On Intuitionistic fuzzy relation, MSc Research Project, Faculty of Science and Technology, Universiti Kebangsaan Malaysia, 2010.

[26] K. T. Atanassov, Intuitionistic fuzzy sets, vol. 35 of Studies in Fuzziness and Soft Computing, Physica, Heidelberg, Germany, 1999, Theory and applications.

[27] F. Hausdorff, Set Theory, Translated from the German by John R. Aumann et al, Chelsea, New York, NY, USA, 1962. 
[28] Y. Yang and F. Chiclana, "Consistency of 2D and 3D distances of intuitionistic fuzzy sets," Expert Systems With Applications, vol. 39, no. 10, pp. 8665-8670, 2012.

[29] M. C. Wu and T. Y. Chen, "The ELECTRE multicriteria analysis approach based on Atanassov's intuitionistic fuzzy sets," Expert Systems with Applications, vol. 38, no. 10, pp. 12318-12327, 2011.

[30] E. Szmidt and J. Kacprzyk, "Group decision making under intuitionistic fuzzy preference relations," in Proceedings of the Information Processing and Management of Uncertainty (IPMU '98), La Sorbonne, Paris, France, 1998.

[31] E. Szmidt and J. Kacprzyk, "Analysis of agreement in a group of experts via distances between intuitionistic fuzzy preferences," in Proceedings of the Information Processing and Management of Uncertainty (IPMU '02), pp. 1859-1865, Annecy, Paris, France, 2002.

[32] E. Szmidt and J. Kacprzyk, "Evaluation of agreement in a group of experts via distances between intuitionistic fuzzy sets," in Proceedings of international IEEE symposium: Intelligent Systems, pp. 166-170, Varna, Bulgaria, 2002.

[33] E. Szmidt and J. Baldwin, "New similarity measures for intuitionistic fuzzy set theory and mass assignment theory," in Proceedings of the 7th International Conference on Intuitionistic Fuzzy Sets, vol. 9, no. 3, pp. 60-76, 2003.

[34] E. Szmidt and J. Kacprzyk, "Entropy for intuitionistic fuzzy set theory and mas assignment theory," Notes on Intuitionistic Fuzzy Sets, vol. 10, no. 3, pp. 15-28, 2004.

[35] E. Szmidt and J. Kacprzyk, "Assigning the parameters for intuitionistic fuzzy sets," Notes on Intuitionistic Fuzzy Sets, vol. 11, no. 6, pp. 1-12, 2005.

[36] E. Szmidt and J. Kacprzyk, "Intuitionistic fuzzy sets in group decision making," Notes on Intuitionistic Fuzzy Sets, vol. 2, pp. 15-32, 1996.

[37] E. Szmidt and J. Kacprzyk, "Remarks on some applications of intuitionistic fuzzy sets in decision making," Notes on Intuitionistic Fuzzy Sets, vol. 2, no. 3, pp. 22-31, 1996.

[38] E. Szmidt and J. Kacprzyk, "Distances between intuitionistic fuzzy sets," Fuzzy Sets and Systems, vol. 114, no. 3, pp. 505-518, 2000.

[39] E. Szmidt and J. Kacprzyk, "Entropy for intuitionistic fuzzy sets," Fuzzy Sets and Systems, vol. 118, no. 3, pp. 467-477, 2001.

[40] E. Szmidt and J. Kacprzyk, "An intuitionistic fuzzy set based approach to intelligent data analysis (an application to medical diagnosis)," in Recent Advances in intelligent Paradigms and Applications, A. Abraham, L. Jain, and J. Kacprzyk, Eds., pp. 5770, Springer, 2002.

[41] L. Tran and L. Duckstein, "Comparison of fuzzy numbers using a fuzzy distance measure," Fuzzy Sets and Systems, vol. 130, no. 3, pp. 331-341, 2002. 


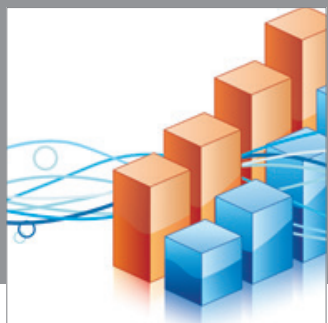

Advances in

Operations Research

mansans

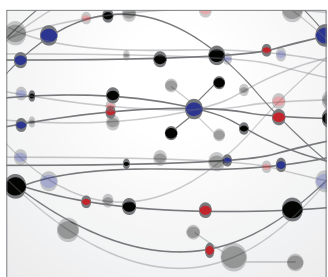

The Scientific World Journal
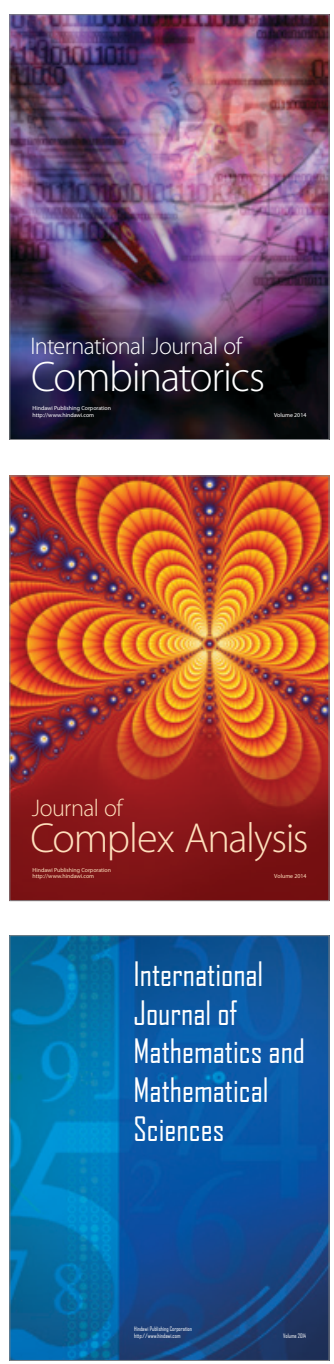
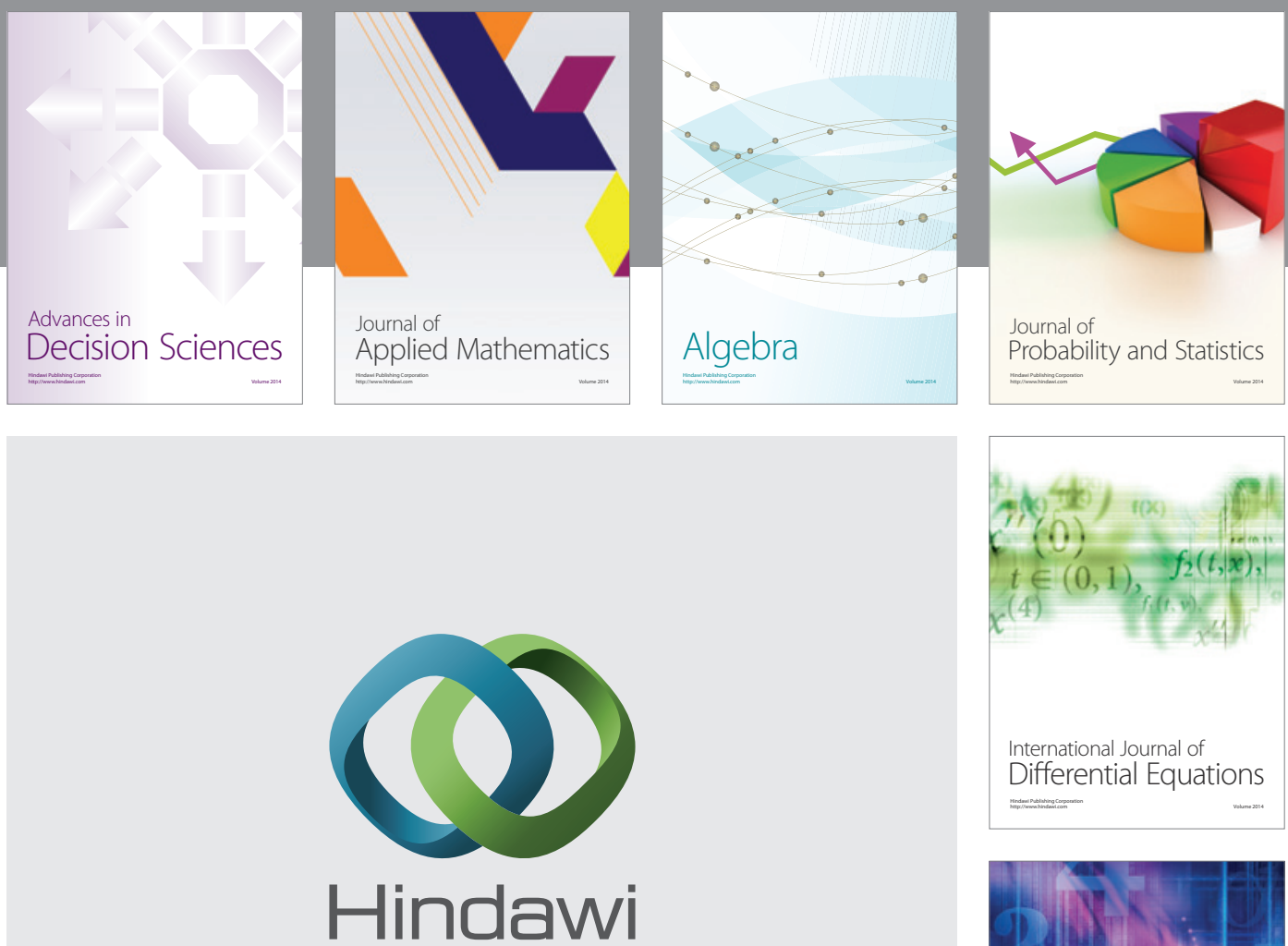

Submit your manuscripts at http://www.hindawi.com
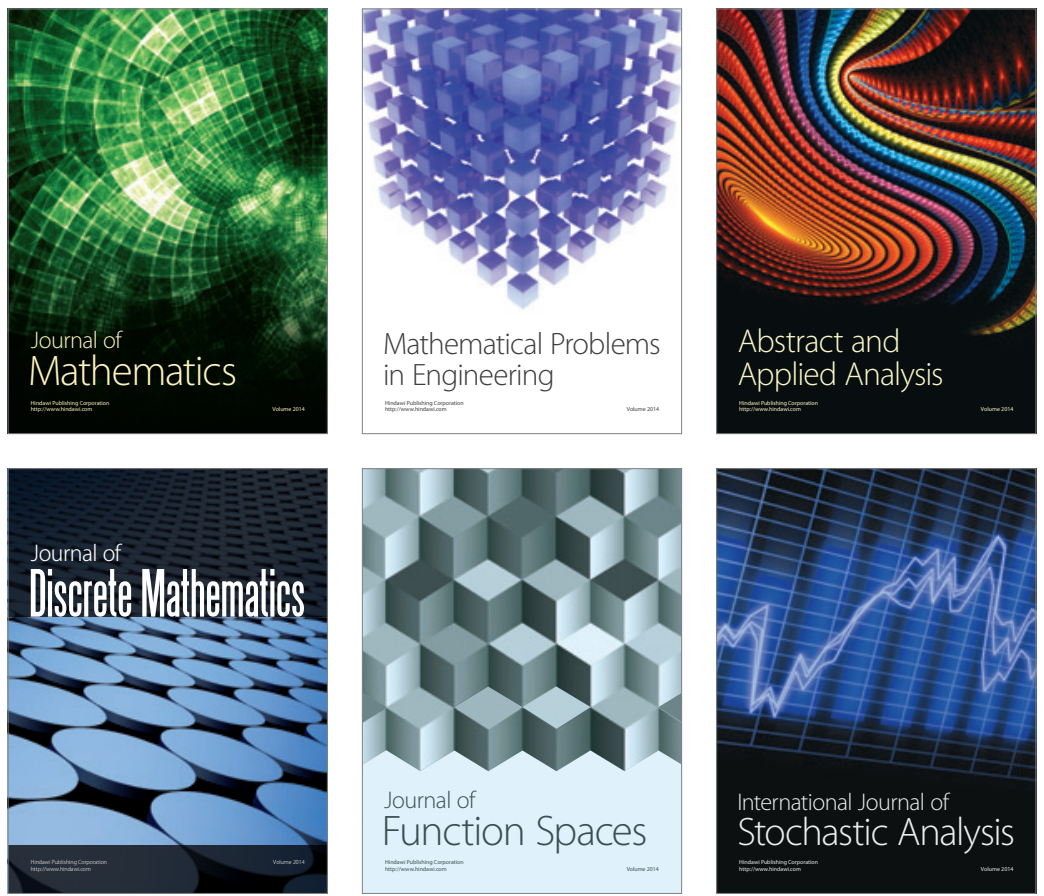

Journal of

Function Spaces

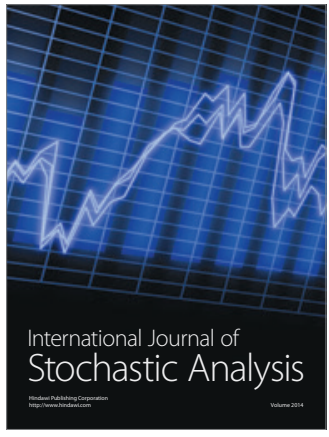

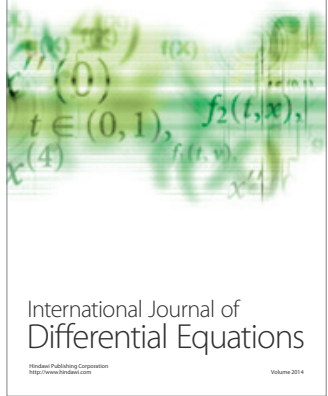
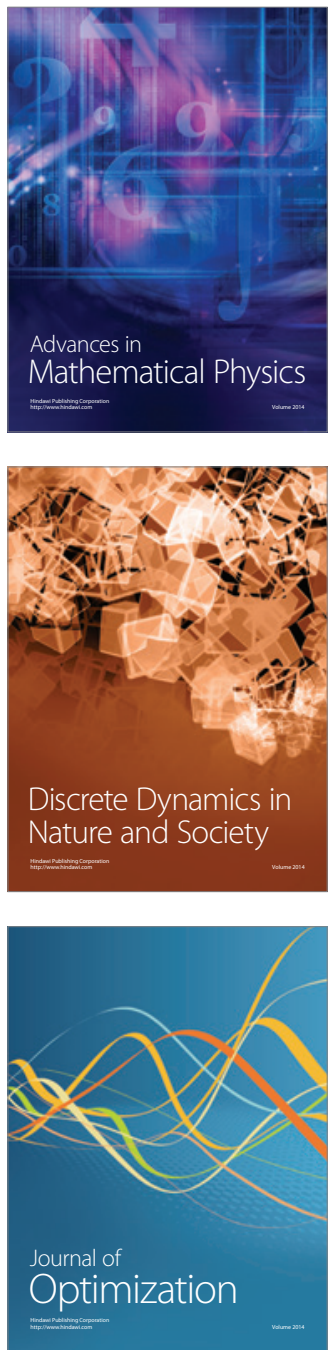